\title{
Assessing the quality of life and well-being of older adults with physical and cognitive impairments in a German- speaking setting: A systematic review of validity and utility of assessments
}

\section{Die Erfassung von Lebensqualität und Wohlbefinden älterer Menschen mit psychischen und kognitiven Einschränkungen: ein systematisches Literaturreview zur Validität und Praktikabilität deutschsprachiger Assessments}

\author{
Thomas Ballmer ${ }^{\star}{ }^{\star}$, Franziska Wirz ${ }^{2}$, Brigitte E. Gantschnig ${ }^{1,3}$ \\ ${ }^{1} Z$ ürcher Hochschule für angewandte Wissenschaften \\ Departement Gesundheit, Institut für Ergotherapie, 8400 \\ Winterthur, Schweiz \\ *thomasmichael.ballmer@zhaw.ch \\ 'Zentrum für medizinische Bildung, 3014 Bern, Schweiz \\ ${ }^{3}$ Universitätsklinik für Rheumatologie, Immunologie und \\ Allergologie, Universitätsspital (Inselspital) und Universität \\ Bern, 3010 Bern, Switzerland \\ Received 22 May 2019, accepted 9 October 2019
}

\begin{abstract}
Background: For health professionals working with older adults with physical and cognitive impairments, improving or maintaining clients' quality of life and well-being is of crucial importance. The aim of this study was to evaluate validity and utility of assessments of quality of life and well-being in German suitable for this group of clients.

Methods: In an initial literature search, we identified potentially viable assessments based on existing systematic reviews. We then conducted a systematic literature search in the databases Medline, CINAHL, and PsycINFO using keywords related to validity, utility, client group, and German. Assessments for which sufficient evidence was found were evaluated regarding their validity and utility when used with older adults with physical and cognitive impairments.

Results: For 14 of 27 initially identified assessments, sufficient evidence was found to evaluate validity and utility with this client group. WHOQOL-BREF, WHOQOL-OLD, WHO-5, EUROHIS-QOL 8, SF-36, SF-12, EQ-5D, NHP, SEIQOL-DW, SWLS, PANAS, DQOL, QOL-AD, and QUALIDEM were evaluated based on 82 studies. Of these, WHOQOL-BREF, WHO-5, SF-36, SF-12, EQ-5D, NHP, QUALIDEM, QOL-AD and DQOL are presented here.

Conclusion: Assessments differed widely in the way they operationalized quality of life/well-being, use of self-evaluation or evaluationby-proxy, and amount of available evidence for their validity and utility. On the basis of our results in regard to the assessments' validity, utility, and appropriateness of operationalization of quality of life/well-being to the client group, three assessments were recommended for use: WHOQOL-BREF for self-evaluation, QUALIDEM for evaluation-by-proxy in case of severe dementia, and EQ-5D for cost-utility analyses.
\end{abstract}

\begin{abstract}
Hintergrund: Für Gesundheitsfachpersonen, die mit älteren Menschen mit physischen und kognitiven Einschränkungen arbeiten, ist die Verbesserung der Lebensqualität und des Wohlbefindens ihrer Klientinnen und Klienten ein wichtiges Ziel. In dieser Studie wurden Validität und Praktikabilität deutschsprachiger Assessments zur Erfassung von Lebensqualität und Wohlbefinden in der Anwendung mit dieser Klientengruppe beurteilt.

Methode: In einer ersten Literaturrecherche wurden in bestehenden Übersichtsarbeiten potenziell geeignete Assessments identifiziert. Für diese wurden in den Datenbanken MEDLINE, CINAHL und PsycINFO eine systematische Suche mit geeigneten Keywords zu den Bereichen „Validität“, „Praktikabilität“, „ältere Menschen“ und „Deutschsprachigkeit“ durchgeführt. Assessments, für welche Evidenz identifiziert werden konnte, wurden bezüglich Validität und Praktikabilität in der Anwendung mit älteren Menschen mit physischen und kognitiven Einschränkungen beurteilt.

Resultate: Für 14 von 27 identifizierten Assessment wurde im Rahmen der systematischen Literaturrecherche genügend Evidenz gefunden, um sie bezüglich Validität und Praktikabilität zu beurteilen. Die Assessments WHOQOL-BREF, WHOQOL-OLD, WHO-5, EUROHIS-QOL 8, SF-36, SF-12, EQ-5D, NHP, SEIQOL-DW, SWLS, PANAS, QUALIDEM, QOL-AD und DQOL wurden anhand von 82 Studien beurteilt. WHOQOL-BREF, WHO-5, SF-36, SF-12, EQ-5D, NHP, QUALIDEM, QOL-AD und DQOL werden in dieser Arbeit näher betrachtet.

Schlussfolgerung: Grundsätzlich unterscheiden sich die 14 Assessments stark bezüglich der Operationalisierung von Lebensqualität/ Wohlbefinden, der Art der Beurteilung (Selbst- oder Fremdbeurteilung) und dem Ausmaß verfügbarer Evidenz. Im Spezifischen empfehlen wir folgende Assessments zur Erfassung von Lebensqualität und Wohlbefinden von älteren Menschen mit physischen und kognitiven Einschränkungen: WHOQOL-BREF zur Selbstbeurteilung, QUALIDEM zur Fremdbeurteilung von Menschen mit schwerer Demenz und zusätzlich den EQ-5D bei Projekten mit gesundheitsökonomischen Fragestellungen.
\end{abstract}




\section{Keywords}

Quality of Life - Well-Being - Assessment - Older Adults - Evaluation - Physical Impairment - Cognitive Impairment

\section{Keywords}

Lebensqualität - Wohlbefinden - Assessment - ältere Menschen - Evaluation - physische Einschränkungen - kognitive Einschränkungen

\section{EINLEITUNG}

In den nächsten Jahren wird die ältere Bevölkerung im deutschsprachigen Raum stark wachsen. Dies betrifft besonders die über 80-Jährigen, deren Zahl laut Prognosen in Deutschland, der Schweiz und Österreich zwischen 2015 und 2045 von ca. 5,5 auf ca.10,9 Millionen steigen wird (Bundesamt für Statistik, 2015; Statistik Austria, 2018; Statistisches Bundesamt, 2015). Ein großer Teil der älteren Bevölkerung lebt mit chronischen Erkrankungen, die mit körperlichen und kognitiven Einschränkungen einhergehen. Zwischen solchen Einschränkungen und subjektiv wahrgenommener Lebensqualität besteht allerdings kein linearer Zusammenhang - Lebensqualität und Wohlbefinden können trotz Einschränkungen subjektiv als hoch eingeschätzt werden (Albrecht \& Devlieger, 1999). Daher wird die Erhaltung und Verbesserung von Lebensqualität und Wohlbefinden in der Bevölkerung zunehmend als eine Aufgabe der Gesundheitsversorgung angesehen (Bundesamt für Gesundheit, 2013; Bundesministerium für Arbeit, Soziales, Gesundheit und Konsumentenschutz, 2019).

Die Begriffe «Lebensqualität» und «Wohlbefinden» werden heutzutage oft verwendet, jedoch selten definiert oder klar voneinander abgegrenzt (Hammell, 2006; Post, 2014). Obwohl Konsensdefinitionen fehlen, wird Lebensqualität meist als subjektiv erlebtes, multidimensionales Konzept beschrieben (Post, 2014), wobei diese Dimensionen unterschiedlich definiert werden, aber oft körperliche und psychische Gesundheit, soziale Beziehungen und Alltagsbewältigung umfassen. Die verbreitetste Definition von subjektivem Wohlbefinden beschreibt dieses als eine Kombination aus allgemeiner Lebenszufriedenheit, Zufriedenheit mit einzelnen Lebensbereichen (z. B. Arbeit oder Familienleben), dem Vorhandsein von positiven Gefühlen und einem geringen Ausmaß von negativen Gefühlen (Diener, 2000). Die definitorische Abgrenzung dieser Begriffe ist nicht Gegenstand dieses Beitrags, sondern die Identifizierung relevanter Assessments zu diesen beiden Konstrukten. Die beiden Begriffe werden im Rahmen dieser Arbeit weitgehend synonym verwendet, wobei deren Operationalisierung in den einzelnen Assessments mit im Fokus steht.

Wenn die Verbesserung oderErhaltung von Lebensqualität und Wohlbefinden ein Ziel der Gesundheitsversorgung ist, müssen Gesundheitsfachpersonen die Möglichkeit haben, diese standardisiert zu erfassen. Auch für den Nachweis der Wirksamkeit von Interventionen im Rahmen klinischer Studien wird die Erfassung von Lebensqualität empfohlen (Glasgow, Magid, Beck, Ritzwoller \& Estabrooks, 2005; Tunis, Stryer \& Clancy, 2003). Gesundheitsfachpersonen benötigen daher Assessments zur Erfassung von Lebensqualität und Wohlbefinden, welche sich sowohl für den Einsatz in der Praxis als auch in der Forschung eignen.

Es gibt eine Vielzahl von krankheitsspezifischen und generischen Assessments, was die Entscheidung für ein Instrument herausfordernd machen kann (Dronavalli \& Thompson,2015). Beider AuswahlvonAssessmentssollten sowohl ihre Validität im Einsatz mit einer bestimmten Klientengruppe als auch ihre Praktikabilität für Praxis und Forschung beachtet werden. „Validität“ bezeichnet den Grad, in dem die Interpretation der Ergebnisse eines Assessments im Rahmen seiner geplanten Verwendung von Theorie und wissenschaftlicher Evidenz gestützt wird (American Educational Research Association, American Psychological Association, National Council on Measurement in Education [AERA, APA \& NCME], 2014). Die Beurteilung von Validität stützt sich daher auf verschiedene Quellen von Evidenz: a) Inhaltliche Validität (i. e. Konstruktvalidität), b) Antwortprozesse (Reliabilität), c) innere Struktur (i. e. interne Konsistenz), d) Beziehung zu anderen Variablen (i. e. konkurrente Validität) und e) Konsequenzen der Bewertung (AERA, APA, \& NCME, 2014). Als „Praktikabilität“ wird mit Fawcett (2013) die allgemeine Brauchbarkeit eines Assessment definiert, die anhand von Kosten, Zeitund Materialaufwand, Akzeptanz bei der Zielgruppe, Ausbildungsaufwand, Benutzerfreundlichkeit für Anwendende, Verfügbarkeit und Verständlichkeit für die Zielgruppe beurteilt wird. Da eine Konsensdefinition für Lebensqualität und Wohlbefinden fehlt, muss untersucht werden, wie diese Konzepte in den verschiedenen Assessments operationalisiert werden.

Das Ziel dieser Arbeit ist es, deutschsprachige Assessments zur Erfassung von Lebensqualität und Wohlbefinden zu identifizieren und zu beurteilen:

a) Welche Bereiche erfassen Erhebungsinstrumente zur Lebensqualität bzw. Wohlbefinden?

b) Sind sie valide im Einsatz mit älteren Menschen mit körperlichen und/oder kognitiven Einschränkungen?

c) Sind sie praktikabel in Praxis und/oder Forschung? 


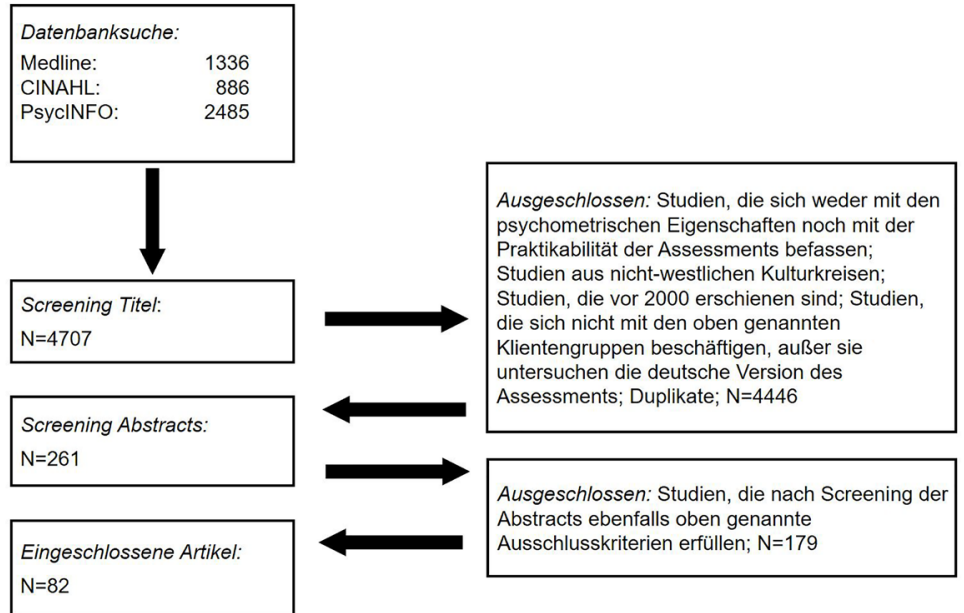

Abbildung 1: Datenbankrecherche, Screening und Auswahl der Studien für die 14 eingeschlossenen Assessments

\section{METHODE}

Angesichts der Vielzahl verfügbarer Assessments zur Erfassung von Lebensqualität und Wohlbefinden identifizierten wir in einer ersten Literaturrecherche bestehende Übersichtsarbeiten (Daig \& Lehmann, 2007; Dronavalli \& Thompson, 2015; Koller et al., 2009; Moniz-Cook et al., 2008; Oppikofer, 2008), um potenziell geeignete Assessments auszuwählen. Kriterien für die Auswahl waren das Vorliegen einer deutschsprachigen Version sowie entweder eine generische Ausrichtung oder eine spezifische Ausrichtung auf ältere Menschen mit körperlichen und/oder kognitiven Einschränkungen. 27 Assessments ergaben sich aus dieser Vorauswahl (siehe Tabelle 1).

Anschließend wurde eine systematische Literaturrecherche in den Datenbanken MEDLINE, CINAHL und PsycINFO durchgeführt. Anhand von einzeln sowie durch die Boole'schen Operatoren AND und OR kombinierten Stich- und Schlagwörtern (siehe Tabelle 2) wurde nach Studien in englischer oder deutscher Sprache gesucht, welche Validität und Praktikabilität der identifizierten Assessments in Bezug auf a) die deutschsprachige Version und/oder b) die Anwendung mit älteren Menschen mit körperlichen und/ oder kognitiven Einschränkungen untersuchen.

Die Titel der gefundenen Studien wurden daraufhin auf ihre inhaltliche Relevanz hin begutachtet. Anschließend wurden anhand der Abstracts weitere nicht relevante Studien ausgeschlossen (siehe Abbildung 1).

Studien aus nicht-westlichen Kulturkreisen sowie vor dem Jahr 2000 erschienene Studien wurden ebenfalls ausgeschlossen, da es kulturell und historisch bedingte Unterschiede gibt, wie Menschen ihre Lebensqualität definieren (Carr \& Higginson, 2001).
Assessments, für die nicht mindestens eine Studie zur deutschsprachigen Version und mindestens eine Studie zur relevanten Klientengruppe gefunden wurde, wurden ausgeschlossen (siehe Tabelle 1). Da die Positive and Negative Affect Scale (Watson, Clark \& Tellegen, 1988) häufig gemeinsam mit der Satisfaction with Life Scale (Diener, Emmons, Larsen \& Griffin, 1985) eingesetzt wird, wurde sie eingeschlossen, obwohl keine aktuellen Studien mit der relevanten Klientengruppe identifiziert werden konnten. In diesem Fall wurden ausnahmsweise auch Studien mit Stichproben aus der gesunden älteren Allgemeinbevölkerung miteingeschlossen.

\section{RESULTATE}

\section{Bereiche von Lebensqualität und Wohlbefinden}

Die untersuchten Assessments unterscheiden sich stark darin, anhand welcher Bereiche sie die Konzepte „Lebensqualität“ und „Wohlbefinden“ operationalisieren. Tabelle 3 vermittelt einen Überblick über die von den 14 analysierten Assessments erfassten Bereiche von Lebensqualität und Wohlbefinden.

\section{Validität und Praktikabilität}

Für 14 der 27 auf der Basis bestehender Übersichtsarbeiten identifizierten, potenziell geeigneten Assessments wurde mindestens eine Studie zur deutschsprachigen Version und mindestens eine Studie zur relevanten Klientengruppe gefunden (siehe Tabelle 1). $\mathrm{Zu}$ diesen 14 Assessments wurden insgesamt 82 relevante Studien identifiziert. Im Rahmen dieser Arbeit werden die neun Assessments behandelt, für die mindestens fünf 
Tabelle 1: Auf der Basis von Übersichtsarbeiten identifizierte, potenziell geeignete Assessments

\begin{tabular}{|c|c|c|c|c|}
\hline Assessment & Autoren & $\begin{array}{l}\text { Eingeschlossen in } \\
\text { Literaturrecherche }\end{array}$ & $\begin{array}{l}\text { Anzahl } \\
\text { Studien }\end{array}$ & $\begin{array}{l}\text { ausgewählte } \\
\text { Studien }\end{array}$ \\
\hline $\begin{array}{l}\text { Dartmouth Cooperative Functional Assess- } \\
\text { ment Charts (COOP/WONCA)) }\end{array}$ & $\begin{array}{l}\text { (Van Weel, Konig-Zahn, Touw-Otten, Van } \\
\text { Duijn \& Meyboom-de Jong, 1995) }\end{array}$ & & & \\
\hline Dementia Quality Of Life (DQOL) & $\begin{array}{c}\text { (Brod, Anita L. Stewart, Sands \& Walton, } \\
\text { 1999) }\end{array}$ & $\checkmark$ & 5 & $\checkmark$ \\
\hline EUROHIS-QOL 8 & (Power, 2003) & $\checkmark$ & 3 & \\
\hline EuroQOL (EQ-5D) & (EuroQol-Group, 1990) & $\checkmark$ & 15 & $\checkmark$ \\
\hline Flourishing Scale (FS) & (Diener et al., 2010) & & & \\
\hline Fragebogen zur Lebenszufriedenheit (FLZ) & (Henrich \& Herschbach, 2000) & & & \\
\hline $\begin{array}{l}\text { Health-related Quality of Life Instrument for } \\
\text { Dementia (DEMQOL) }\end{array}$ & (Smith et al., 2005) & & & \\
\hline Nottingham Health Profile (NHP) & $\begin{array}{l}\text { (Hunt, McKenna, McEwen, Williams \& Papp, } \\
\text { 1981) }\end{array}$ & $\checkmark$ & 5 & $\checkmark$ \\
\hline Patient Generated Index (PGI) & $\begin{array}{c}\text { (Ruta, Garratt, Leng, Russell \& MacDonald, } \\
\text { 1994) }\end{array}$ & & & \\
\hline Personal Wellbeing Index (PWI) & $\begin{array}{l}\text { (Cummins, Eckersley, Pallant, Van Vugt \& } \\
\text { Misajon, 2003) }\end{array}$ & & & \\
\hline $\begin{array}{l}\text { Positive and Negative Affect Schedule } \\
\text { (PANAS) }\end{array}$ & (Watson, Clark \& Tellegen, 1988) & $\checkmark$ & 3 & \\
\hline $\begin{array}{l}\text { Quality of Life in Alzheimer's Disease (QOL- } \\
\qquad A D)\end{array}$ & (Logsdon, Gibbons, McCurry \& Teri, 1999) & $\checkmark$ & 8 & $\checkmark$ \\
\hline $\begin{array}{l}\text { Quality of Life in Late Stage Dementia } \\
\text { (QUALID) }\end{array}$ & (Weiner et al., 2000) & & & \\
\hline QUALIDEM & $\begin{array}{c}\text { (Ettema, Dröes, de Lange, Mellenbergh \& } \\
\text { Ribbe, 2007a, 2007b) }\end{array}$ & $\checkmark$ & 9 & $\checkmark$ \\
\hline Quality of Life Questionnaire (QOL-Q) & (Schalock, Hoffman \& Keith, 1993) & & & \\
\hline Quality of Life Scale (QOLS) & (Flanagan, 1978) & & & \\
\hline Quality of Wellbeing Scale (QWB) & (Kaplan, Anderson \& Ganiats, 1993) & & & \\
\hline Satisfaction With Life Scale (SWLS) & (Diener, Emmons, Larsen \& Griffin, 1985) & $\checkmark$ & 4 & \\
\hline $\begin{array}{l}\text { Schedule for the Evaluation of Individual } \\
\text { Quality of Life (SEIQoL) }\end{array}$ & $\begin{array}{l}\text { (O’Boyle, McGee, Hickey, O’Malley \& Joyce, } \\
\text { 1992) }\end{array}$ & $\checkmark$ & 4 & \\
\hline Short Form 12 (SF-12) & (Ware, Kosinski \& Keller, 1996) & $\checkmark$ & 9 & $\checkmark$ \\
\hline Short Form 36 (SF-36) & (Ware \& Sherbourne, 1992) & $\checkmark$ & 8 & $\checkmark$ \\
\hline Short Form 8 (SF-8) & (Ware, Kosinski, Dewey \& Gandek, 2001) & & & \\
\hline Sickness Impact Profile (SIP) & (Gilson et al., 1975) & & & \\
\hline Spitzer-Index (SI) & (Spitzer et al., 1981) & & & \\
\hline WHO-Five Well-Being Index (WHO-5) & $\begin{array}{c}\text { (World Health Organization Regional Office } \\
\text { for Europe, 1998) }\end{array}$ & $\checkmark$ & 5 & $\checkmark$ \\
\hline $\begin{array}{c}\text { World Health Organization Quality of Life- } \\
\text { BREF (WHOQOL-BREF) }\end{array}$ & (WHOQOL-Group, 1998) & $\checkmark$ & 5 & $\checkmark$ \\
\hline $\begin{array}{l}\text { World Health Organization Quality of Life- } \\
\text { OLD (WHOQOL-OLD) }\end{array}$ & (Power, Quinn \& Schmidt, 2005) & $\checkmark$ & 4 & \\
\hline
\end{tabular}


Tabelle 2. Suchworttabelle Literaturrecherche in den Datenbanken Medline, CINAHL und PsycINFO

\begin{tabular}{c|c|c|c|c}
\hline Stichwörter & $\begin{array}{c}\text { Name und } \\
\text { Kurzform des } \\
\text { Assessments } \\
\text { (z. B. EUROQOL, } \\
\text { EQ-5D*) }\end{array}$ & $\begin{array}{c}\text { validity, reliability, objectivity, } \\
\text { psychometric*, feasibility ; Validität, } \\
\text { Reliabilität, Objektivität, Psychometr*, } \\
\text { Praktikabilität }\end{array}$ & $\begin{array}{c}\text { Elderly, old*, nursing home, dementia, } \\
\text { Alzheimer*, cognitve impairment; } \\
\text { alter*, alt*, Pflegeheim, Altersheim, } \\
\text { Demenz, Alzheimer, kognitive Beein- } \\
\text { trächtigung*, }\end{array}$ & $\begin{array}{c}\text { German*, } \\
\text { Deutsch* }\end{array}$ \\
\hline $\begin{array}{c}\text { MeSH Terms } \\
\text { (Medline) }\end{array}$ & & $\begin{array}{c}\text { PSYCHOMETRICS, REPRODUCIBILITY OF } \\
\text { RESULTS, VALIDATION STUDIES, BIAS }\end{array}$ & $\begin{array}{c}\text { AGED, DEMENTIA, COGNITION DISOR- } \\
\text { DERS, COGNITIVE DYSFUNCTION }\end{array}$ & $\begin{array}{c}\text { GERMANY, } \\
\text { SWITZERLAND, } \\
\text { AUSTRIA }\end{array}$ \\
\hline $\begin{array}{c}\text { CINAHL Subject } \\
\text { Headings }\end{array}$ & & PSYCHOMETRICS, MEASUREMENT \\
ERROR, RELIABILITY AND VALIDITY, & AGED, DEMENTIA, COGNITION DISOR- \\
RsycINFO Thesau- \\
rus
\end{tabular}

Tabelle 3. Bereiche von Lebensqualität und Wohlbefinden

\begin{tabular}{|c|c|c|c|c|c|c|c|c|c|c|c|c|c|c|}
\hline & $\begin{array}{l}\text { WHOQOL- } \\
\text { BREF }\end{array}$ & $\begin{array}{l}\text { QOL- } \\
\text { AD }\end{array}$ & $\begin{array}{l}\text { EUROHIS- } \\
\text { QOL } 8\end{array}$ & NHP & $\begin{array}{l}\text { SF- } \\
36\end{array}$ & $\begin{array}{l}\text { SF- } \\
12\end{array}$ & $\begin{array}{l}\text { WHOQOL- } \\
\text { OLD }\end{array}$ & $\begin{array}{c}\text { QUALI- } \\
\text { DEM }\end{array}$ & $\mathrm{DQOL}$ & $\begin{array}{c}\text { WHO- } \\
5\end{array}$ & $\begin{array}{l}E Q- \\
5 D\end{array}$ & SWLS & PANAS & SEIQOL-DW \\
\hline $\begin{array}{l}\text { Psychische } \\
\text { Gesundheit } \\
\text { und Gefühle }\end{array}$ & $\checkmark$ & $\checkmark$ & $\checkmark$ & $\checkmark$ & $\checkmark$ & $\checkmark$ & & $\checkmark$ & $\checkmark$ & $\checkmark$ & $\checkmark$ & & $\checkmark$ & $\begin{array}{c}\text { Von } \\
\text { Befragten } \\
\text { ausgewählt }\end{array}$ \\
\hline $\begin{array}{l}\text { Alltägliche } \\
\text { Tätigkeiten }\end{array}$ & $\checkmark$ & $\checkmark$ & $\checkmark$ & $\checkmark$ & $\checkmark$ & $\checkmark$ & $\checkmark$ & $\checkmark$ & & & $\checkmark$ & & & $\begin{array}{c}\text { Von } \\
\text { Befragten } \\
\text { ausgewählt }\end{array}$ \\
\hline $\begin{array}{c}\text { Soziale } \\
\text { Beziehungen }\end{array}$ & $\checkmark$ & $\checkmark$ & $\checkmark$ & $\checkmark$ & $\checkmark$ & $\checkmark$ & $\checkmark$ & $\checkmark$ & $\checkmark$ & & & & & $\begin{array}{c}\text { Von } \\
\text { Befragten } \\
\text { ausgewählt }\end{array}$ \\
\hline $\begin{array}{l}\text { Sinn und } \\
\text { Bedeutung }\end{array}$ & $\checkmark$ & $\checkmark$ & $\checkmark$ & $\checkmark$ & & & $\checkmark$ & & $\checkmark$ & $\checkmark$ & & $\checkmark$ & & $\begin{array}{c}\text { Von } \\
\text { Befragten } \\
\text { ausgewählt }\end{array}$ \\
\hline $\begin{array}{l}\text { Energie/ } \\
\text { Vitalität }\end{array}$ & $\checkmark$ & $\checkmark$ & $\checkmark$ & $\checkmark$ & $\checkmark$ & $\checkmark$ & & & & $\checkmark$ & & & & $\begin{array}{c}\text { Von } \\
\text { Befragten } \\
\text { ausgewählt }\end{array}$ \\
\hline $\begin{array}{l}\text { Identität und } \\
\text { Selbstbild }\end{array}$ & $\checkmark$ & $\checkmark$ & $\checkmark$ & & & & $\checkmark$ & $\checkmark$ & $\checkmark$ & & & $\checkmark$ & & $\begin{array}{c}\text { Von } \\
\text { Befragten } \\
\text { ausgewählt }\end{array}$ \\
\hline $\begin{array}{l}\text { Körperliche } \\
\text { Gesundheit }\end{array}$ & $\checkmark$ & $\checkmark$ & $\checkmark$ & & $\checkmark$ & $\checkmark$ & & & & & & & & $\begin{array}{c}\text { Von } \\
\text { Befragten } \\
\text { ausgewählt }\end{array}$ \\
\hline $\begin{array}{l}\text { Schmerzen/ } \\
\text { Beschwerden }\end{array}$ & $\checkmark$ & & & $\checkmark$ & $\checkmark$ & $\checkmark$ & & & & & $\checkmark$ & & & $\begin{array}{c}\text { Von } \\
\text { Befragten } \\
\text { ausgewählt }\end{array}$ \\
\hline $\begin{array}{l}\text { Umwelt und } \\
\text { Lebens- } \\
\text { bedingungen }\end{array}$ & $\checkmark$ & $\checkmark$ & $\checkmark$ & & & & & & & & & $\checkmark$ & & $\begin{array}{c}\text { Von } \\
\text { Befragten } \\
\text { ausgewählt }\end{array}$ \\
\hline Schlaf & $\checkmark$ & & & $\checkmark$ & & & & & & $\checkmark$ & & & & $\begin{array}{c}\text { Von } \\
\text { Befragten } \\
\text { ausgewählt }\end{array}$ \\
\hline $\begin{array}{l}\text { Sinnesfunk- } \\
\text { tionen und } \\
\text { Gedächtnis }\end{array}$ & $\checkmark$ & $\checkmark$ & & & & & $\checkmark$ & & & & & & & $\begin{array}{c}\text { Von } \\
\text { Befragten } \\
\text { ausgewählt }\end{array}$ \\
\hline
\end{tabular}

Anmerkungen: Mit Häkchen markierte Felder: Assessment enthält Fragen bzw. Items zum jeweiligen Bereich (basierend auf Bereichsbezeichnung im Assessment selbst oder, wenn keine solche vorliegt, auf Beurteilung des Inhalts der Fragen bzw. Items durch die Autorenschaft dieser Studie); dunkelgrau markierte Felder: Assessment enthält keine Fragen bzw. Items zum jeweiligen Bereich; hellgrau markierte Felder: Erfasste Bereiche werden von Befragten selber definiert. 


\begin{tabular}{|c|c|}
\hline Beschreibung & $\begin{array}{l}\text { Autoren/Entwicklung: Kurzversion des WHOQOL-100, der als kulturübergreifendes Instrument zur Erfassung der } \\
\text { Lebensqualität entwickelt wurde (Brähler \& Gunzelmann, 2002) } \\
\text { Erhobenes Konstrukt: Lebensqualität } \\
\text { Erhebungsform: Selbstbeurteilungsfragebogen } \\
\text { Items: } 24 \text { Fragen in vier Bereichen; zwei allgemeine Fragen } \\
\text { Bewertung: fünfstufige Skala (Qualität, Ausmaß, Häufigkeit, Zufriedenheit) } \\
\text { Auswertung: Berechnung von vier Bereichswerten } \\
\text { Erfasster Zeitraum: Letzte zwei Wochen }\end{array}$ \\
\hline Praktikabiltität & $\begin{array}{l}\text { Kosten: kostenlos } \\
\text { Zeitaufwand: durchschnittlich } 15 \text { Minuten (Lucas-Carrasco et al., 2011c) } \\
\text { Material: Fragebogen } \\
\text { Akzeptanz: allgemein gut, bei Frage zum Sexualleben eingeschränkt (Kalfoss et al., 2008; Skevington et al., 2004) } \\
\text { Ausbildungsaufwand: k. A. Eine deutsche Version des Manuals ist verfügbar (Angermeyer et al., 2000) } \\
\text { Benutzerfreundlichkeit: k. A. } \\
\text { Verfügbarkeit: Bei Unterzeichnung einer Lizenzierungsvereinbarung frei erhältlich unter http://www.who.int/mental_ } \\
\text { health/publications/whoqol/en/ } \\
\text { Verständlichkeit: k. A. } \\
\text { Andere Sprachversionen: in über } 30 \text { Sprachen erhältlich (Topp et al., 2015) }\end{array}$ \\
\hline Validität & $\begin{array}{l}\text { Inhaltliche Validität: Angemessenheit Vier-Bereiche Struktur umstritten (Skevington et al., 2004; Kalfoss et al., 2008) } \\
\text { Antwortprozesse: TRR für den Bereich „physische Gesundheit“ akzeptabel (ICC =0.71), andere Bereiche ICC zwischen 0,51 } \\
\text { und 0,61 beurteilt (Lucas-Carrasco et al., 2011a) } \\
\text { Innere Struktur: Akzeptable interne Konsistenz (Crohnbach's } \alpha \text { zwischen 0,76 und 0,88) aller Bereiche der deutschen } \\
\text { Version (Skevington et al., 2004); Studien mit anderssprachigen Versionen bestätigen dies für alle Bereiche außer soziale } \\
\text { Beziehungen ( } \alpha \text { zwischen 0,54 und 0,67; Kalfoss et al., 2008; Lucas-Carrasco et al., 2011a) } \\
\text { Beziehung zu anderen Variablen: Korrelation physische Gesundheit mit BI } r=0,46 \text { (Lucas-Carrasco et al., 2011a); Bereiche } \\
\text { physische und psychische Gesundheit mit entsprechenden Bereichen des SF-12 } r=0,66-0,78 \text { bzw. 0,45-0,62 (Kalfoss et } \\
\text { al., 2008); soziale Beziehungen und entsprechender Bereich des SF-12 } r=0,29-0,32 \text { (Kalfoss et al., 2008); physische und } \\
\text { psychische Gesundheit sowie Umwelt mit GDS ( } r=-0,73 ; r=-0,83 ; r=-0,83 \text { ) und körperlichen Symptomen ( } r=-0,67 ; r=-0,61 \text {; } \\
\text { r=-0,60; Naumann \& Byrne, 2004) } \\
\text { Konsequenzen der Bewertung: k. A. }\end{array}$ \\
\hline $\begin{array}{l}\text { Gefundene } \\
\text { Studien } \\
\text { (Autoren/ } \\
\text { Sprache/ } \\
\text { Population/ } \\
\text { Sample/Alter } \\
\text { (Durchschnitt } \\
\text { und SD)) }\end{array}$ & $\begin{array}{l}\text { Aigner et al., 2006; Deutsch; Menschen mit chronischen Schmerzen; N=100; } 46,4(11,4) \\
\text { Skevington et al., 2004; versch. (Deutsch); gesunde und kranke Menschen; } \mathrm{N}=11830(2408) ; 45(16) \\
\text { Lucas-Carrasco et al., 2011a; Spanisch; Menschen mit leichter bis mittlerer Demenz (MMSE über 10), die zhause wohnen; } \\
\mathrm{N}=104 ; 78,6(7,2) \\
\text { Kalfoss et al., 2008; Englisch/Norwegisch; Menschen über 60, zu Hause oder im Pflegeheim lebend; N=192/ N=469; 72,8 } \\
(8,5) / 75,7 \text { (8,0) } \\
\text { Naumann et al., 2004; Englisch; Ältere Menschen mit Depression; N=39; } 79,5(5,1)\end{array}$ \\
\hline
\end{tabular}

Anmerkungen: $B I=$ Barthel Index; GDS = Geriatric Depression Scale; ICC = Intraclass Correlation; $N=$ Anzahl; $r$ = Spearman Correlation Coefficient; $S D=$ Standardabweichung; $T R R=$ Test -Retest -Reliabilität

Studien gefunden werden konnten (siehe Tabelle 1). Die Resultate der Recherche werden für diese Assessments kurz beschrieben. Ausführlicher sind die Resultate in den Tabellen 4 bis 12 dargestellt.

\section{World Health Organisation Quality of Life Assessment-Bref (WHOQOL-BREF)}

Der WHOQOL-BREF wurde von der Weltgesundheitsorganisation (WHO) als Assessment zur Erfassung von Lebensqualität entwickelt (WHOQOLGroup, 1998). Er umfasst 24 Fragen zu den Bereichen körperliche und psychische Gesundheit, soziale Beziehungen und Umwelt sowie zwei generelle Fragen (zu Lebensqualität bzw. Gesundheit). Diese werden von den Befragten auf einer fünfstufigen Skala bewertet. Der WHOQOL-BREF wurde anhand von fünf Studien mit 39 bis 11830 Teilnehmenden beurteilt. Er wird allgemein als praktikabel eingeschätzt und lässt sich mit Personen mit bis zu mittelschwerer Demenz einsetzen (Kalfoss, Low \& Molzahn, 2008; Lucas-Carrasco, Skevington, Gómez-Benito, Rejas \& March, 2011; Skevington, Lotfy \& O'Connell, 2004). Eine Frage zum Sexualleben wurde von relativ vielen Teilnehmenden nicht beantwortet (Kalfoss et al., 2008; Skevington et al., 2004). Die Validität des WHOQOL-BREF wird von den gefundenen Studien weitgehend gestützt (Aigner et al., 2006; Kalfoss et al., 2008; Lucas-Carrasco, Skevington, et al., 2011; Naumann \& Byrne, 2004; Skevington et al., 2004). Es liegt auch eine Kurzversion mit acht Fragen vor, der European Health Interview Survey-Quality of Life 8 (EUROHIS-QOL 8; Power, 2003). Zu diesem besteht allerdings im untersuchten Bereich noch wenig Evidenz. 


\begin{tabular}{|c|c|}
\hline Beschreibung & $\begin{array}{l}\text { Autoren/Entwicklung: durch WHO basierend auf bestehenden Assesments entwickelt (Bonsignore et al., 2001) } \\
\text { Erfasstes Konstrukt: Wohlbefinden } \\
\text { Erhebungsform: Selbstbeurteilungsfragebogen } \\
\text { Items: fünf positiv formulierte, kurze Aussagen } \\
\text { Bewertung: sechsstufige Skala (Häufigkeit) } \\
\text { Auswertung: Summenscore } \\
\text { Erfasster Zeitraum: letzte zwei Wochen }\end{array}$ \\
\hline Praktikabiltität & $\begin{array}{l}\text { Kosten: kostenlos } \\
\text { Zeitaufwand: k. A. } \\
\text { Material: Fragebogen } \\
\text { Akzeptanz: gut bei Pflegeheimbewohnern (Allgaier et al., 2013) } \\
\text { Ausbildungsaufwand: k. A. } \\
\text { Benutzerfreundlichkeit: wenig fehlende Antworten (Brähler et al., 2007; Schneider et al., 2010) } \\
\text { Verfügbarkeit: frei verfügbar } \\
\text { Verständlichkeit: k. A. } \\
\text { Andere Sprachversionen: in über } 30 \text { Sprachen erhältlich (Topp et al. 2015) }\end{array}$ \\
\hline Validität & $\begin{array}{l}\text { Inhaltliche Validität: Bestätigung einfaktorielle Struktur (Brähler et al., 2007; Bonsignore et al., 2011); mäßige } \\
\text { Deckeneffekte in der Allgemeinbevölkerung (Brähler et al., 2007) } \\
\text { Antwortprozesse: TRR nur eingeschränkt bestätigt ( } r=0,67 ; \text { Englbrecht et al., 2017); Resultat aber aufgrund } \\
\text { methodologischer Mängel zweifelhaft. } \\
\text { Innere Struktur: angemessene Korrelationen zwischen Items ( } r=0,37-0,79) \text { und zwischen Items und Gesamtwert ( } r=0,52- \\
\text { 0,82; Brähler et al., 2017; Schneider et al., 2010); gute interne Konsistenz mit Crohnbach's } \alpha=0,93 \text { bzw. 0,83 (Allgaier et al., } \\
\text { 2013; Schneider et al., 2010) } \\
\text { Beziehung zu anderen Variablen (Auswahl): Korrelation mit Fragebogen zur Erfassung des körperlichen Wohlbefindens } \\
r=0,56 \text { bis 0,65 (Brähler et al., 2007); mit BDI } r=-0,52 \text { bis -0,75 (Englbrecht et al., 2017; Schneider et al., 2010); mit SOMS } \\
r=0,53 \text { (Brähler et al., 2007); hohe Spezifizität und Sensitivität bei der Identifikation von Personen mit Depression (Allgaier } \\
\text { et al., 2013; Englbrecht et al., 2017; Bonsignore et al., 2001; Schneider et al., 2010) } \\
\text { Konsequenzen der Bewertung: k. A. }\end{array}$ \\
\hline $\begin{array}{l}\text { Gefundene } \\
\text { Studien } \\
\text { (Autoren/ } \\
\text { Sprache/ } \\
\text { Population/ } \\
\text { Sample/Alter } \\
\text { (Durchschnitt } \\
\text { und SD)) }\end{array}$ & $\begin{array}{l}\text { Brähler et al., 2007; Deutsch; Allgemeinbevölkerung; N=2473; 48,1 (-) } \\
\text { Bonsignore et al., 2001; Deutsch; Menschen mit/ohne Depression; N=367; 70,2 (8,0) } \\
\text { Allgaier et al., 2013; Deutsch; Pflegeheimbewohner; } N=92 ; 84,5(-) \\
\text { Schneider et al., 2010; Deutsch; Menschen mit Parkinson; } N=215 ; 68,2(8,9) \\
\text { Englbrecht et al., 2017; Deutsch; Menschen mit Arthritis; N=262; } 59,8(13,2)\end{array}$ \\
\hline
\end{tabular}

Anmerkungen: $B D I=$ Beck Depression Inventory; $I C C=$ Intraclass Correlation; $N=$ Anzahl; $r=$ Spearman Correlation Coefficient; SD = Standardabweichung; SOMS = Screening Somatoforme Störungen; $T R R=$ Test-Retest-Reliabilität

\section{WHO-5 Wohlbefindens-Index}

Der WHO-5 wurde von der WHO (1998) zur Erfassung von Wohlbefinden entwickelt. Fünf Items (positive Gefühle, Entspannung, Energie, Schlafqualität, Interesse am eigenen Alltag) werden von den Befragten auf einer sechsstufigen Skala bewertet (WHO, 1998). Der WHO-5 wurde anhand von fünf Studien mit 92 bis 2473 Teilnehmenden beurteilt. Die Praktikabilität wird als gut eingeschätzt, zur Anwendung mit Menschen mit kognitiven Einschränkungen wurden jedoch keine Angaben gefunden (Allgaier et al., 2013; Brähler, Mühlan, Albani \& Schmidt, 2007; Schneider et al., 2010). Aufgrund der identifizierten Studien kann die Validität des WHO-5 als akzeptabel beurteilt werden (Allgaier et al., 2013; Brähler et al., 2007; Englbrecht et al., 2017; Heun, Bonsignore, Barkow \& Jessen, 2001; Schneider et al., 2010).

\section{Short Form Health Survey 36 (SF-36)}

Mit dem SF-36 (Ware \& Sherbourne, 1992) wird der subjektive Gesundheitszustand mit 36 Fragen in acht Bereichen (körperliche Funktionsfähigkeit, körperliche Rollenfunktion, körperliche Schmerzen, allgemeine Gesundheitswahrnehmung, Vitalität, soziale Funktionsfähigkeit, emotionale Rollenfunktion, psychisches Wohlbefinden) erfasst. Er kann zur Selbstoder Fremdbeurteilung verwendet werden. Die Fragen werden auf zwei- bis sechsstufigen Skalen bewertet. Der SF-36 wurde anhand von acht Studien mit 138 bis 6728 Teilnehmenden beurteilt. Die Praktikabilität wird als gut, bei älteren und/oder schwerkranken Menschen jedoch als reduziert eingeschätzt (Bullinger et al., 2003; Novella et al., 2001). Die identifizierten Studien stützen die Validität des SF-36 im untersuchten Bereich größtenteils; bei Menschen mit stärkeren kognitiven Einschränkungen ist diese aber reduziert (Buchcik, Westenhöfer, Fleming 


\begin{tabular}{|c|c|}
\hline Beschreibung & $\begin{array}{l}\text { Autoren/Entwicklung: Im Rahmen der Medical Outcome Study entwickelt (Ware \& Sherbourne, 1992). } \\
\text { Erfasstes Konstrukt: subjektiver Gesundheitszustand } \\
\text { Erhebungsform: Selbstbeurteilungsfragebogen oder Interview } \\
\text { Items: } 36 \text { Fragen in acht Bereichen } \\
\text { Bewertung: zwei- bis sechsstufige Skalen (Qualität, Intensität, Zustimmung, Frequenz) } \\
\text { Auswertung: Berechnung von acht Bereichswerten und zwei Gesamtwerten (psychische/ körperliche Gesundheit) } \\
\text { Erfasster Zeitraum: Letzte vier Wochen bzw. letzte Woche }\end{array}$ \\
\hline Praktikabiltität & $\begin{array}{l}\text { Kosten: kostenpflichtig } \\
\text { Zeitaufwand: 7-15 Minuten (Bullinger et al., 2000), Menschen mit Demenz: durchschnittlich ca. } 19 \text { Minuten (Novella et al., } \\
\text { 2001) } \\
\text { Material: Fragebogen (Interview- oder Selbstbeurteilungsversion) } \\
\text { Akzeptanz: allgemein gut, bei älteren und schwer erkrankten Menschen reduziert (Bullinger et al., 2000; Novella et al., } \\
\text { 2001) } \\
\text { Ausbildungsaufwand: k. A. } \\
\text { Benutzerfreundlichkeit: k. A. } \\
\text { Verfügbarkeit: über lizenzierenden Verlag } \\
\text { Verständlichkeit: k. A. } \\
\text { Andere Sprachversionen: laut lizenzierender Firma 170+ Sprachversionen }\end{array}$ \\
\hline Validität & $\begin{array}{l}\text { Inhaltliche Validität: Acht-Bereiche-Struktur weitgehend bestätigt (Bullinger et al., 2003; Maurischat et al., 2006; Morfeld } \\
\text { et al., 2005; Novella et al., 2001); Gebrauch der Gesamtwerte nicht empfohlen (Buchcik et al., 2017; Maurischat et al., } \\
\text { 2008). } \\
\text { Antwortprozesse: TRR der Bereichswerte eingeschränkt bis gut bei Menschen mit Demenz mit MMSE >15 (ICC=0,53-0,90), } \\
\text { deutlich niedriger bei MMSE <15 (ICC=0,17 bis 0,81; Novella et al., 2001). } \\
\text { Innere Struktur: gute interne Konsistenz aller Dimensionen (Crohnbach's } \alpha=0,66 \text { bis 0,93; Bullinger et al., 2003; } \\
\text { Gunzelmann et al., 2006; Morfeld et al., 2005) bzw. bei Menschen mit Demenz in fünf Bereichen } \alpha>0,7 \text { (Novella et al., } \\
\text { 2001); signifikant geringere interne Konsistenz bei Menschen mit kognitiven Beeinträchtigungen in drei Dimensionen } \\
\text { ( } p=0,0003-0,04 ; \text { Seymour et al., 2001). } \\
\text { Beziehung zu anderen Variablen (Auswahl): Korrelationen mit EQ-5D körperliche Subskala } r=0,51 \text { bis 0,70, psychische } \\
\text { Subskala } r=0,42 \text { bis 0,58 (Bullinger et al., 2003); Bereich Körperliche Funktionsfähigkeit mit FIM r=0,53 (Seymour et al., } \\
\text { 2001); alle Bereiche und Subskalen mit CES-D } r=-0,41 \text { bis -0,62 (Bullinger et al., 2003) } \\
\text { Konsequenzen der Bewertung: k. A. }\end{array}$ \\
\hline $\begin{array}{l}\text { Gefundene } \\
\text { Studien } \\
\text { (Autoren/ } \\
\text { Sprache/ } \\
\text { Population/ } \\
\text { Sample/Alter } \\
\text { (Durchschnitt } \\
\text { und SD)) }\end{array}$ & $\begin{array}{l}\text { Buchcik et al., 2017; Deutsch/Polnisch/Türkisch; ältere türkische/polnische Migranten und Deutsche; N=304; 68,3(6,9) } \\
\text { Bullinger et al., 2003; Deutsch; Rehabilitationspatienten (Metaanalyse); N=6728; 45,85 (-) bis 57,73 (-) } \\
\text { Frosch et al., 2001; Deutsch; Männer mit Prostata-Krebs/-Hyperplasie, Darm- oder Analkrebs; N=277; 63,55 (-) bis 68,48 } \\
\text { (-) } \\
\text { Gunzelmann et al., 2006; Deutsch; ältere Allgemeinbevölkerung; N=690; k. A. (60-93 Jahre) } \\
\text { Maurischat et al., 2006; Deutsch; Menschen mit Spondylitis ankylosans; N=436; 44,3(10) } \\
\text { Morfeld et al., 2005; Deutsch; Allgemeinbevölkerung; N=2043; 48,9 (18,1) } \\
\text { Novella et al., 2001; Französisch; Menschen mit Demenz; N=138; 82,7(-) } \\
\text { Seymour et al., 2001; Englisch; ältere Rehabilitationspatienten mit/ohne kognitive Beeinträchtigungen; N=314; 79,7(-) }\end{array}$ \\
\hline
\end{tabular}

Anmerkungen: $C E S-D=$ Center for Epidemiological Studies-Depression Scale ; FIM = Functional Independence Measure; ICC = Intraclass Correlation; $r=$ Spearman Correlation Coefficient SD = Standardabweichung; TRR = Test-Retest-Reliabilität

\& Martin, 2017; Bullinger et al., 2003; Frosch et al., 2001; Gunzelmann, Albani, Beutel \& Brähler, 2006; Maurischat, Ehlebracht-Konig, Kuhn \& Bullinger, 2006; Morfeld, Bullinger, Nantke \& Brähler, 2005; Novella et al., 2001; Seymour et al., 2001).

\section{Short Form Health Survey 12 (SF-12)}

Der SF-12 ist eine Kurzversion des SF-36 mit 12 Fragen (Ware, Kosinski \& Keller, 1996). Er wurde anhand von neun Studien mit 60 bis 4526 Teilnehmenden beurteilt. Praktikabilität und Validität werden als gut eingeschätzt, Angaben zu Menschen mit kognitiven Einschränkungen fehlen jedoch (Haibel et al., 2004; Jakobsson, 2007; Jakobsson, Westergren, Lindskov \& Hagell, 2012;
Maurischat et al., 2006; Maurischat, Herschbach, Peters \& Bullinger, 2008; Morfeld, Dietsche, Bürger \& Koch, 2003; Muller-Nordhorn, 2004; Scholz et al., 2013; Wirtz, Morfeld, Glaesmer \& Brähler, 2018). Mit dem SF-12 beziehungsweise dem SF-36 erfasste Daten lassen sich mittels des SF-6D (Brazier, Usherwood, Harper \& Thomas, 1998) in gesundheitsökonomisch auswertbare Quality-Adjusted Life Years (QALY) umrechnen.

\section{EuroQol-5D (EQ-5D)}

Der EQ-5D wurde als Assessment zur Erfassung der gesundheitsbezogenen Lebensqualität entwickelt, welches sich als Grundlage zur Berechnung von QALY eignet (EuroQol, 1990). Das Assessment 


\begin{tabular}{|c|c|}
\hline Beschreibung & $\begin{array}{l}\text { Autoren/Entwicklung: Kurzform des SF-36 } \\
\text { Erfasstes Konstrukt: subjektiver Gesundheitszustand } \\
\text { Erhebungsform: Selbstbeurteilungsfragebogen oder Interview } \\
\text { Items: } 12 \text { Fragen in zwei Bereichen } \\
\text { Bewertung: zwei- bis sechsstufige Skalen (Qualität, Intensität, Zustimmung, Frequenz) } \\
\text { Auswertung: Berechnung zweier Gesamtwerte (körperliche/ psychische Gesundheit) } \\
\text { Erfasster Zeitraum: letzte vier Wochen bzw. letzte Woche }\end{array}$ \\
\hline Praktikabiltität & $\begin{array}{l}\text { Kosten: kostenpflichtig } \\
\text { Zeitaufwand: zwei Minuten (Maurischat et al., 2006), keine Angaben für Menschen mit kognitiven Beeinträchtigungen } \\
\text { Material: Fragebogen (Interview- oder Selbstbeurteilungsversion) } \\
\text { Akzeptanz: Wenig fehlende Werte in den meisten Studien (Jakobsson, 2007; Jakobsson et al., 2012; Maurischat et al., } \\
\text { 2006; Maurischat et al., 2008; Müller-Nordhorn, 2004) } \\
\text { Ausbildungsaufwand: k. A. } \\
\text { Benutzerfreundlichkeit: k. A. } \\
\text { Verfügbarkeit: über lizenzierenden Verlag } \\
\text { Verständlichkeit: k. A. } \\
\text { Andere Sprachversionen: laut lizenzierender Firma zahlreiche Versionen }\end{array}$ \\
\hline $\begin{array}{l}\text { Gefundene } \\
\text { Studien } \\
\text { (Autoren/ } \\
\text { Sprache/ } \\
\text { Population/ } \\
\text { Sample/Alter } \\
\text { (Durchschnitt } \\
\text { und SD)) }\end{array}$ & $\begin{array}{l}\text { Haibel et al., 2004; Deutsch; Menschen mit Ankolysierender Spondylitis; N=60; 38,6 (-) } \\
\text { Maurischat et al., 2006; Deutsch; Menschen mit rheumatischen Erkrankungen; } N=545 ; 44,5(9,9) \\
\text { Maurischat et al., 2008; Deutsch; Menschen mit Diabetes; N=343; 51,9(7,8) } \\
\text { Morfeld et al., 2003; Deutsch; Menschen mit Erkrankungen des Bewegungs- und Stützapparates; N=1176; 49,9(11,4) } \\
\text { Müller-Nordhorn, 2004; Deutsch; Menschen mit mit Herz-Krankheiten; N=2441; ? } 60 \text { (10), ? } 65(10) \\
\text { Scholz et al., 2013; Deutsch; Menschen mit muskuloskelettalen Erkrankungen (in Rehabilitation); N=184; } 59(14,9) \\
\text { Wirtz et al., 2018; Deutsch; Allgemeinbevölkerung; N=2524; } 49(18,3) \\
\text { Jakobsson, 2007; Schwedisch; hochaltrige Allgemeinbevölkerung; N=4278; 83,7(5,7) } \\
\text { Jakobsson et al., 2012; Schwedisch; Hochaltrige Allgemeinbevölkerung (AB), Menschen mit Parkinson (PA) und nach } \\
\text { Schlaganfall (SA); AB: N=4278, PA: N=159, SA: N=89; AB: 83,7[5,7), PA: 70,5 (7,9), SA: 77,2 (6,7); }\end{array}$ \\
\hline
\end{tabular}

Anmerkungen: BASFI = Bath Ankylosing Spondylitis Functional Index; HADS-D/A = Hospital Anxiety and Depression Scale (Dimension Ängstlichkeit); HADS-D/A = Hospital Anxiety and Depression Scale (Dimension Depression); IADL = Instrumental Activities of Daily Life; ICC=Intraclass Correlation; $O D I=$ Oswestry Disability Index; PADL: Personal Activities of Daily Life; $N=$ Anzahl; $S D=$ Standardabweichung

kann zur Selbst- oder Fremdbeurteilung verwendet werden und besteht aus fünf Items (Mobilität, Selbstversorgung, alltägliche Aktivitäten, Schmerzen/ Beschwerden, Angst/Depression). Jedes Item umfasst drei (EQ-5D-3L) beziehungsweise fünf (EQ-5D-5L) Antwortmöglichkeiten (keine, einige oder schwere Probleme im jeweiligen Bereich). Die Antworten werden in einen präferenzbasierten Indexwert zwischen 0 und 1 umgerechnet. Zusätzlich wird der Gesundheitszustand auf einer visuell-analogen Skala beurteilt (Wolfs et al., 2007). Der EQ-5D wurde anhand von 15 Studien mit 64 bis 2469 Teilnehmern beurteilt. Die Praktikabilität wird als gut, bei schwerer Demenz jedoch als eingeschränkt beurteilt (Ankri et al., 2003; Holland, Smith, Harvey, Swift \& Lenaghan, 2004; Karlawish et al., 2008; Kunz, 2010; Naglie et al., 2006; Orgeta, Edwards, Hounsome, Orrell \& Woods, 2015). Die Validität des EQ-5D wird von den gefundenen Studien größtenteils gestützt, ist bei schwerer Demenz aber reduziert (Aguirre et al., 2016; Ankri et al., 2003; Brettschneider et al., 2013; Bryan, Hardyman, Bentham, Buckley \& Laight, 2005; Coucill, Bryan, Bentham, Buckley \& Laight, 2001; DiazRedondo, Rodriguez-Blazquez, Ayala, Martinez-Martin \& Forjaz, 2014; Easton, Milte, Crotty \& Ratcliffe, 2018; Günther, Roick, Angermeyer \& König, 2008; Hinz, Klaiberg, Schumacher \& Brahler, 2003; Holland et al., 2004; Karlawish et al., 2008; Kunz, 2010; Naglie et al., 2006; Orgeta et al., 2015; Sonntag, Konig \& Konnopka, 2015).

\section{Nottingham Health Profile (NHP)}

Das NHP ist ein Assessment zur Erfassung der gesundheitsbezogenen Lebensqualität (Hunt, McKenna, 


\begin{tabular}{|c|c|}
\hline Beschreibung & $\begin{array}{l}\text { Autoren/Entwicklung: EuroQol-Group (1990) } \\
\text { Erhobenes Konstrukt: gesundheitsbezogene Lebensqualität } \\
\text { Erhebungsform: Selbst- und Fremdbeurteilungsversion } \\
\text { Items: fünf Bereiche und eine visuelle Skala zur Beurteilung des subjektiven Gesundheitszustandes } \\
\text { Bewertung: zu jedem Bereich drei Aussagen (keine, einige, schwere Probleme), zutreffendste wird angekreuzt } \\
\text { Auswertung: Indexwert } \\
\text { Erfasster Zeitraum: Erfassungszeitpunkt }\end{array}$ \\
\hline Praktikabiltität & $\begin{array}{l}\text { Kosten: kostenlos für Forschungszwecke, Lizenzierungsgebühr für Einsatz in Praxis (abhängig von Anzahl Klienten); } \\
\text { Zeitaufwand: Fremdbeurteilung durchschnittlich } 2 \text { Minuten, Selbstbeurteilung durchschnittlich } 5 \text { bis } 15 \text { Minuten (Ankri et } \\
\text { al., 2003; Holland et al., 2004; Naglie et al., 2006) } \\
\text { Material: Fragebogen } \\
\text { Akzeptanz: allgemein wenig fehlende Antworten; Antwortrate bei schwerer Demenz deutlich reduziert (Ankri et al., 2003; } \\
\text { Holland et al., 2004; Karlawish et al., 2008; Kunz, 2010; Orgeta et al., 2015) } \\
\text { Ausbildungsaufwand: k. A. } \\
\text { Benutzerfreundlichkeit: k. A. } \\
\text { Verfügbarkeit: online } \\
\text { Verständlichkeit: k. A. } \\
\text { Andere Sprachversionen: in über } 130 \text { Sprachen erhältlich }\end{array}$ \\
\hline Validität & $\begin{array}{l}\text { Inhaltliche Validität: signifikante Korrelationen zwischen allen Bereichen außer Selbstversorgung mit der VAS des EQ-5D } \\
\text { (Ankri et al., 2003) } \\
\text { Antwortprozesse: unterschiedliche Ergebnisse zu IRR ( } k=0,00 \text { bis 0,61, ICC=0,41 bis 0,72; Ankri et al., 2003; Diaz-Redondo } \\
\text { et al., 2014) und TRR bei Demenz ( } k=0,34 \text { bis 0,59, ICC=0,54 bis 0,83; Ankri et al., 2003; Karlawish et al., 2008; Naglie et al., } \\
\text { 2006). TRR bei EQ-VAS Selbstbeurteilung eingeschränkt (ICC=0,08 bis 0,55; Ankri et al., 2003, Naglie et al., 2006) } \\
\text { Innere Struktur: Interne Konsistenz knapp akzeptabel (Crohnbach's } \alpha=0,60 \text { bis 0,66; Aguirre et al., 2016; Diaz-Redondo et } \\
\text { al., 2014) } \\
\text { Beziehung zu anderen Variablen (Auswahl): Selbstbeurteilung: Indexwerte mit MBI } r=0,49 \text { (Easton et al., 2018); mit SF-36 } \\
\text { körperliche Subskala } r=0,57 \text { bis 0,64 (Brettschneider et al., 2016), mit QOL-AD } r=0,40 \text { bis 0,43 (Aguirre et al., 2015); mit } \\
\text { PHQ-9 } r=-0,53 \text { bis }-0,35 \text { (Brettschneider et al., 2016; Hinz et al., 2014); Fremdbeurteilung: Indexwerte mit MBI } r=0,56 \\
\text { (Easton et al., 2018; mit MMSE } r=0,19 \text { bis 0,50 (Wolfs et al., 2007). } \\
\text { Konsequenzen der Bewertung: k. A. }\end{array}$ \\
\hline $\begin{array}{l}\text { Gefundene } \\
\text { Studien } \\
\text { (Autoren/ } \\
\text { Sprache/ } \\
\text { Population/ } \\
\text { Sample/Alter } \\
\text { (Durchschnitt } \\
\text { und SD)) }\end{array}$ & 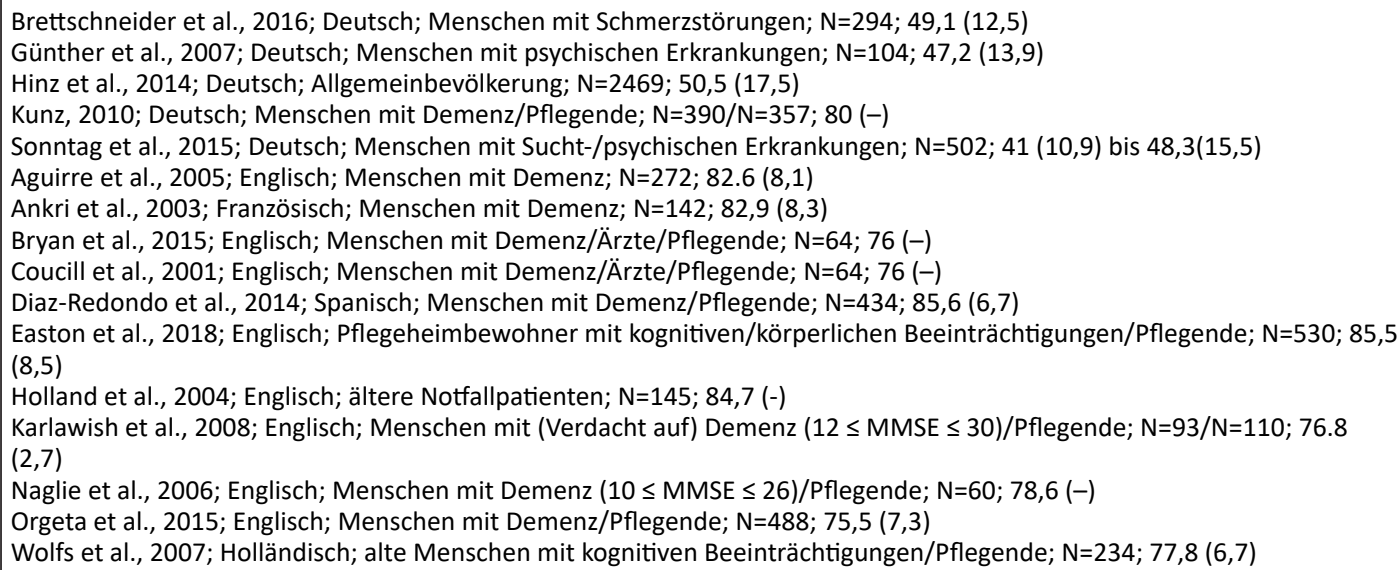 \\
\hline
\end{tabular}

Anmerkungen: ICC = Intraclass Correlation; IRR = Inter-Rater-Reliabilität; $M B I=$ Modified Barthel Index; $M M S E=$ Mini Mental State Examination; $N$ = Anzahl; $P H Q-9=$ Patient Health Questionnaire 9 (Depression); SD = Standardabweichung; TRR = Test-Retest-Reliabilität

McEwen, Williams \& Papp, 1981). Es umfasst 38 Aussagen in sechs Bereichen (Energie, Schmerz, emotionale Reaktion, Schlaf, soziale Isolation, physische Mobilität), die dichotom bewertet werden. Das NHP wurde anhand von fünf Studien mit 99 bis 1966 Teilnehmern beurteilt. Die Praktikabilität ist bei Menschen mit Demenz reduziert. Bei Depression wird das Assessment nicht empfohlen (Baró et al., 2006; Boyer, Novella, Morrone, Jolly \& Blanchard, 2004; Simon, Berwig \& Heinrich, 2013; Tabali et al., 2012).
Die Validität des NHP wird von den identifizierten Studien größtenteils gestützt (Baró et al., 2006; Boyer et al., 2004; Hinz et al., 2003; Simon et al., 2013; Tabali et al., 2012).

\section{QUALIDEM}

Das QUALIDEM (Ettema, Dröes, de Lange, Mellenbergh \& Ribbe, 2007a, 2007b) dient zur Fremdbeurteilung der Lebensqualität von Pflegeheimbewohnern mit 


\begin{tabular}{|c|c|}
\hline Beschreibung & $\begin{array}{l}\text { Autoren/Entwicklung: Hunt et al. (1981) } \\
\text { Erhobenes Konstrukt: gesundheitsbezogene Lebensqualität } \\
\text { Erhebungsform: Selbstbeurteilungs-Fragebogen oder Interview } \\
\text { Items: } 38 \text { negativ formulierte Aussagen } \\
\text { Bewertung: dichotom («trifft zu»/«trifft nicht zu») } \\
\text { Auswertung: Umrechnung in Bereichswerte (0-100) und Gesamtwert } \\
\text { Erfasster Zeitraum: Erfassungszeitpunkt }\end{array}$ \\
\hline Praktikabiltität & $\begin{array}{l}\text { Kosten: k. A. } \\
\text { Zeitaufwand: durchschnittlich } 13 \text { Minuten (Boyer et al., 2004; Tabali et al., 2011) bis } 40 \text { Minuten (Simon et al., 2013) } \\
\text { Material: Fragebogen } \\
\text { Akzeptanz: Antwortquote ohne Demenz } 76 \text { \% bis } 98 \text { \%; leichte bis mittelschwere Demenz } 49 \text { \% bis } 86 \% \text {; schwere Demenz } \\
6 \text { \% bis } 9 \% \text { (Baró et al., 2006; Tabali et al., 2012). } \\
\text { Ausbildungsaufwand: k. A. } \\
\text { Benutzerfreundlichkeit: k. A. } \\
\text { Verfügbarkeit: über Urheberrechtsinhaber } \\
\text { Verständlichkeit: k. A. } \\
\text { Andere Sprachversionen: in zahlreiche Sprachen übersetzt }\end{array}$ \\
\hline $\begin{array}{l}\text { Gefundene } \\
\text { Studien } \\
\text { (Autoren/ } \\
\text { Sprache/ } \\
\text { Population/ } \\
\text { Sample/Alter } \\
\text { (Durchschnitt } \\
\text { und SD)) }\end{array}$ & $\begin{array}{l}\text { Hinz et al., 2003; Deutsch; Allgemeinbevölkerung; } \mathrm{N}=1966 \text {; ? } 50,2(16,3) \text {, ? } 49,9(17,2) \\
\text { Tabali et al., 2012; Deutsch; Pflegeheimbewohner mit/ohne Demenz; } N=286 ; 83,6(8,8) \\
\text { Simon et al., 2013; Deutsch; Pflegeheimbewohner mit/ohne Demenz; } N=145 ; 81,8(10,3) \\
\text { Baró et al., 2006; Spanisch; Ältere Patienten mit körperlichen oder kognitiven Beeinträchtigungen; } N=134 ; 79,8(7,3) \\
\text { Boyer et al., 2004; Französisch; Patienten mit leichter bis mittelschwerer Demenz; } N=99 ; 81,8(8,2)\end{array}$ \\
\hline
\end{tabular}

Anmerkungen: GBB-24 = Giessener Beschwerdebogen; HADS-A = Hospital Anxiety and Depression Scale (Dimension Ängstlichkeit); HADS-D = Hospital Anxiety and Depression Scale (Dimension Depression); ICC = Intraclass Correlation; MFI-20 = Multidimensional Fatigue Inventory; $r=$ Spearman Correlation Coefficient; $N=$ Anzahl; $S D=$ Standardabweichung; TRR = Test-Retest-Reliabilität

Demenz. Es besteht aus 37 beobachtbaren Items in neun Bereichen (Pflegebeziehung, Positiver Affekt, Negativer Affekt, Unruhiges/angespanntes Verhalten, Positives Selbstbild, Soziale Beziehungen, Soziale Isolation, Sich zu Hause fühlen, Etwas zu tun haben). Die Items werden auf einer siebenstufigen Skala bewertet. Das QUALIDEM wurde anhand von neun Studien mit 91 bis 2158 Teilnehmenden beurteilt. Seine Praktikabilität wird als gut beurteilt (Bouman et al., 2011; Dichter, Bartholomeyczik, Nordheim, Achterberg \& Halek, 2011; Gräske et al., 2014). Die Studien stützen die Validität des QUALIDEM, im Bereich Beziehung zu anderen Variablen besteht jedoch noch wenig Evidenz (Arons et al., 2018; Bouman et al., 2011; Dichter et al., 2011,
2013, 2014; Dichter, Wolschon, Meyer \& Kopke, 2016; Ettema, Dröes, de Lange, Mellenbergh \& Ribbe, 2007b, 2007a; Gräske et al., 2014).

\section{Quality of Life - Alzheimer's Disease (QOL-AD)}

Der QOL-Ad ist ein Assessment zur Erfassung der Lebensqualität bei Menschen mit AlzheimerDemenz, welches Selbst- und Fremdeinschätzung kombiniert (Logsdon, Gibbons, McCurry \& Teri, 1999). Er beinhaltet 13 Fragen, welche die Bereiche Körperlicher Gesundheitszustand, Energie, Stimmung, Wohnverhältnisse, Gedächtnis, Ehe/Partnerschaft, Freunde, Person insgesamt, Fähigkeit, Aufgaben in 


\begin{tabular}{|c|c|}
\hline Beschreibung & $\begin{array}{l}\text { Autoren/Entwicklung: Ettema et al. (2007a, 2007b) basierend auf Dröes' (1991) Adaption-Coping Modell } \\
\text { Erhobenes Konstrukt: Lebensqualität bei Demenz } \\
\text { Erhebungsform: Fremdbeurteilung } \\
\text { Items: } 38 \text { Verhaltensweisen in neun Bereichen } \\
\text { Bewertung: vier- bzw. siebenstufige Skala } \\
\text { Auswertung: Bereichswerte (Summen-Scores) } \\
\text { Erfasster Zeitraum: letzte zwei Wochen }\end{array}$ \\
\hline Praktikabiltität & $\begin{array}{l}\text { Kosten: kostenlos } \\
\text { Zeitaufwand: } 6 \text { bis } 15 \text { Minuten (Boumann et al., 2011; Dichter et al., 2011; Gräske et al., 2014) } \\
\text { Material: Fragebogen } \\
\text { Akzeptanz: k. A. } \\
\text { Ausbildungsaufwand: k. A. } \\
\text { Benutzerfreundlichkeit: größtenteils als gut beurteilt (Dichter et al., 2011; Gräske et al., 2014) } \\
\text { Verfügbarkeit: Assessment und Benutzerhandbuch frei erhältlich } \\
\text { Verständlichkeit: größtenteils als gut beurteilt (Dichter et al., 2011; Gräske et al., 2014) } \\
\text { Andere Sprachversionen: Holländisch und Englisch (Dichter et al., 2016b) }\end{array}$ \\
\hline Validität & $\begin{array}{l}\text { Inhaltliche Validität: Bereichsstruktur zum Teil bestätigt (Gräske et al., 2014) zum Teil nicht bestätigt (Dichter et al., 2011) } \\
\text { Antwortprozesse: IRR für Originalversion eingeschränkt (ICC=0,28 bis 0,79; Dichter et al., 2014; Ettema et al., 2007b), für } \\
\text { deutsche Version/bei Verwendung eines Handbuchs größtenteils gut bis sehr gut (ICC=0,64 bis 0,96; Dichter et al., 2016a); } \\
\text { TRR gut bei ICC=0,70 bis 0,89 (Dichter et al., 2014, Ettema et al., 2007b) } \\
\text { Innere Struktur: nicht eindeutige Ergebnisse zu einzelnen Bereichen (Arons et al., 2018; Bouman et al., 2011; Dichter et al., } \\
\text { 2011; Dichter et al., 2016a; Ettema et al., 2007b; Gräske et al., 2014) } \\
\text { Beziehung zu anderen Variablen (Auswahl): Korrelation mit einem studieneigenen Fragebogen zur Lebensqualität } r=-0,11 \\
\text { bis 0,44 durch Familienangehörige beurteilt, } r=-0,20 \text { bis 0,44 durch Pflegefachpersonen beurteilt. Korrelation mit } \\
\text { selbstbeurteilten COOP/WONCA-Charts r=-0,24 bis 0,1. Korrelation aller Bereiche außer Etwas zu tun haben mit CSDD } \\
r=-0,52 \text { bis -0,28, Soziale Beziehungen und Positiver Affekt mit asozialem Verhalten (BIP; r=-0,75 bzw. -0,64; alle Angaben } \\
\text { Ettema et al., 2007a). Keine Unterschiede nach Alter und Geschlecht (Gräske et al., 2014) } \\
\text { Konsequenzen der Bewertung: k. A. }\end{array}$ \\
\hline $\begin{array}{l}\text { Gefundene } \\
\text { Studien } \\
\text { (Autoren/ } \\
\text { Sprache/ } \\
\text { Population/ } \\
\text { Sample/Alter } \\
\text { (Durchschnitt } \\
\text { und SD)) }\end{array}$ & $\begin{array}{l}\text { Dichter et al. 2016; Deutsch; Pflegeheimbewohner mit leichter bis schwerer Demenz; } N=91 ; 84,1(9,4)-84,7(8,3) \\
\text { Dichter et al. 2014; Deutsch; Pflegeheimbewohner mit leichter bis schwerer Demenz; } N=108 ; 85,2(7)-84,2(9) \\
\text { Dichter et al. 2013; Deutsch; Pflegeheimbewohner mit leichter bis schwerer Demenz; } N=643 ; 85,8(8)-84,5(8,2) \\
\text { Dichter et al. 2011; Deutsch; Pflegeheimbewohner mit leichter bis schwerer Demenz; } N=486 ; 84(9)-86(8) \\
\text { Gräske et al. 2014; Deutsch; Bewohner von Demenz-Wohngemeinschaften mit leichter bis schwerer Demenz; N=104; } 79(-) \\
\text { Aron et al. 2018; Holländisch; Pflegeheimbewohner mit leichter bis schwerer Demenz; } N=2158 ; 85,2(7,6) \\
\text { Bouman et al. 2011; Holländisch; Pflegeheimbewohner mit leichter bis schwerer Demenz; } N=973 ; 82,9(6,8)-85(5,7) \\
\text { Ettema et al. 2007a; Holländisch; Pflegeheimbewohner mit leichter bis schwerer Demenz; } N=202 ; 84,7(6,9) \\
\text { Ettema et al. 2007b; Holländisch; Pflegeheimbewohner mit leichter bis schwerer Demenz; } N=238 ; 84,7(6,9)\end{array}$ \\
\hline
\end{tabular}

Anmerkungen: $B I P=$ Behavioral assessment scale for Intramural Psychogeriatrics; COOP/WONCA = Dartmouth Cooperative Functional Assessment Charts (höhere Werte = niedrigere Lebensqualität); CSDD = Cornell Scale for Depression in Dementia; ICC = Intraclass Correlation; IRR =Inter-RaterReliabilität; $N=$ Anzahl; $r=$ Spearman Correlation Coefficient; $S D=$ Standardabweichung; TRR = Test-Retest-Reliabilität

und um das Haus zu erledigen, Fähigkeit, etwas zum Vergnügen zu machen, Geld und Leben insgesamt erfassen. Die Fragen werden sowohl von der Person mit Demenz selbst als auch von einer betreuenden Person auf einer vierstufigen Skala bewertet (Wolak et al., 2009). Der QOL-AD wurde anhand von acht Studien mit 94 bis 277 Teilnehmenden beurteilt. Seine Praktikabilität wird weitgehend als gut, bei schwerer Demenz jedoch als eingeschränkt beurteilt (Gräske et al., 2014; Hoe, Katona, Roch \& Livingston, 2005; LeonSalas, Logsdon, Olazaran, Martinez-Martin \& The MsuAdru, 2011; Wolak et al., 2009; Wolak-Thierry et al., 2015). Bei Fremdbeurteilungen fehlten im QOL-AD oft Bewertungen im Bereich Geld, möglicherweise, weil die Beurteilenden nicht über die finanzielle Situation der Beurteilten informiert waren (Dichter et al., 2018). Die Validität des QOL-AD wird von den gefundenen Studien weitgehend gestützt (Barrios et al., 2013; Dichter, Wolschon, Schwab, Meyer \& Köpke, 2018; Gräske et al., 2014; Hoe et al., 2005; Hylla, Schwab, Isfort, Halek \& Dichter, 2016; Leon-Salas et al., 2011; Wolak et al., 2009; Wolak-Thierry et al., 2015).

\section{Dementia Quality of Life Instrument (DQOL)}

Der DQOL ist ein krankheitsspezifisches Assessment zur Erfassung der Lebensqualität von Menschen mit bis zu mittelschwerer Demenz (Brod, Stewart, Sands \& Walton, 1999). Er wird als Interview durchgeführt und besteht aus 29 Fragen zu den Bereichen positive Gefühle, negative Gefühle, Zugehörigkeitsgefühle, Selbstwert und Sinn für Ästhetik sowie einer Frage zur allgemeinen Lebensqualität, die auf einer fünfstufigen Skala beantwortet werden (Voigt-Radloff et al., 2012). 


\begin{tabular}{|c|c|}
\hline Beschreibung & $\begin{array}{l}\text { Autoren/Entwicklung: auf Grundlage von Literatur zu Lebensqualität bei Demenz und anderen krankheitsspezifischen } \\
\text { Erfassungsinstrumenten entwickelt (Logsdon et al., 1999) } \\
\text { Erhobenes Konstrukt: Lebensqualität bei Demenz (selbst- und fremdbewertet) } \\
\text { Erhebungsform: Selbst- und Fremdbeurteilungsfragebogen } \\
\text { Items: } 13 \text { Fragen } \\
\text { Bewertung: fünfstufige Skala (Qualität, Ausmaß, Häufigkeit, Zufriedenheit) } \\
\text { Auswertung: Summen-Scores (kombinierter Wert kann errechnet werden) } \\
\text { Erfasster Zeitraum: Erfassungszeitpunkt }\end{array}$ \\
\hline Validität & $\begin{array}{l}\text { Inhaltliche Validität: Ergebnisse zur Eindimensionalität uneinheitlich (Hylla et al., 2016; Wolak et al., 2009) } \\
\text { Antwortprozesse: gute TRR QOL-AD patient (ICC=0,83 bis 0,92) und QOL-AD composite (ICC=0,82 bis 0,89; Barrios et al., } \\
\text { 2013; Leon-Salas et al., 2011; Wolak et al., 2009; Wolak-Thierry et al., 2015). Für QOL-AD proxy TRR gut (ICC=0,7 bis 0,92; } \\
\text { Barrios et al., 2013; Wolak et al., 2009; Wolak-Thierry et al., 2015) bzw. ungenügend (ICC=0,52; Leon-Salas et al., 2011). } \\
\text { Schwache bis mittlere IRR (ICC=0,65; Dichter et al., 2018) } \\
\text { Innere Struktur: gute interne Konsistenz (Crohnbach's } \alpha=0,74 \text { bis 0,9; Barrios et al., 2013; Gräske et al. 2015; Hoe et al., } \\
\text { 2005; Hylla et al., 2016; Leon-Salas et al., 2011; Wolak et al., 2009; Wolak-Thierry et al., 2015) } \\
\text { Beziehung zu anderen Variablen (Auswahl): Korrelationen QOL-AD patient mit SWLS r=0,54 (Barrios et al., 2013), mit } \\
\text { DHP-general health r=0,54 (Wolak et al., 2009), mit BI r=0,32 (Leon-Salas et al., 2011), mit DQOL r=0,36 bis 0,63 (Wolak- } \\
\text { Thierry et al., 2015), mit Zarit's Caregiver Burden score r=0,09 (Wolak et al., 2009), mit CSDD r=-0,16 (Leon-Salas et al., } \\
\text { 2011), mit GDS r=-0,7 (Barrios et al., 2013); mit NPI (neuropsychiatrische Symptome) r=-0,14 bis -0,06 (Barrios et al., } \\
\text { 2013; Leon-Salas et al., 2011; Wolak et al., 2009); QOL-AD proxy mit DHP-general health r=0,39 (Wolak et al.,, 2009), mit } \\
\text { BI r=0,55 (Leon-Salas et al., 2011), mit DQOL r=-0,19 bis 0,43 (Wolak-Thierry et al., 2015), mit Zarit's Caregiver Burden } \\
\text { score r=-0,38 (Wolak et al., 2009), mit CSDD r=-0,29 (Leon-Salas et al., 2011), mit GDS r=-0,7 (Barrios et al., 2013), mit NPI } \\
\text { (neuropsychiatrische Symptome) r=-0,28 bis -0,40 (Barrios et al., 2013; Leon-Salas et al., 2011; Wolak et al., 2009); } \\
\text { Konsequenzen der Bewertung: k. A. }\end{array}$ \\
\hline
\end{tabular}

Anmerkungen: $B I=$ Barthel Index; $C S D D=$ Cornell Scale for Depression in Dementia; GDS = Geriatric Depression Scale; ICC = Intraclass Correlation; $I R R=$ Inter-Rater-Reliabilität; $N=$ Anzahl; NPI =Neuropsychiatric Inventory; $r=$ Spearman Correlation Coefficient; $S D=$ Standardabweichung; $S W L S$ = Satisfaction With Life Scale; TRR= Test-Retest-Reliabilität

Der DQOL wurde anhand von fünf Studien mit 67 bis 486 Teilnehmenden beurteilt. Seine Praktikabilität wurde größtenteils als gut eingeschätzt, allerdings war Verständnis und Akzeptanz bei den Befragten teilweise eingeschränkt (Voigt-Radloff et al., 2012; Wolak-Thierry et al., 2015). Seine Validität wurde von den identifizierten Studien größtenteils gestützt (Adler \& Resnick, 2010; Lucas-Carrasco, Gomez-Benito, Rejas \& Brod, 2011; Ready, Ott \& Grace, 2007; Voigt-Radloff et al., 2012; Wolak-Thierry et al., 2015).

\section{DISKUSSION}

Die Ergebnisse dieser Arbeit zeigen, dass es eine Auswahl von praktikablen und validen deutschsprachigen Assessments zur Erhebung von Lebensqualität und Wohlbefinden bei älteren Menschen mit körperlichen und/ oder kognitiven Einschränkungen gibt, die sich bezüglich ihrer Praktikabilität und Validität, der Operationalisierung von Lebensqualität und Wohlbefinden sowie der Art der Bewertung unterscheiden. Diese drei Punkte werden im Folgenden gesondert diskutiert. 


\begin{tabular}{|c|c|}
\hline Beschreibung & $\begin{array}{l}\text { Autoren/Entwicklung: Brod et al. (1999) } \\
\text { Erhobenes Konstrukt: Lebensqualität bei Demenz } \\
\text { Erhebungsform: Interview } \\
\text { Items: } 29 \text { Fragen in fünf Bereichen und eine allgemeine Frage zur Lebensqualität } \\
\text { Bewertung: fünfstufige Skala } \\
\text { Auswertung: Summen-Scores der einzelnen Bereiche } \\
\text { Erfasster Zeitraum: Erfassungszeitpunkt }\end{array}$ \\
\hline Praktikabiltität & $\begin{array}{l}\text { Kosten: k. A. } \\
\text { Zeitaufwand: ca. } 14 \text { Minuten (Wolak-Thierry et al., 2015) } \\
\text { Material: Fragebogen } \\
\text { Akzeptanz: allgemein gut (Voigt-Radloff et al. ,2012; Wolak-Thierry et al. 2015), für Bereich Sinn für Ästhetik teilweise } \\
\text { eingeschränkt (Voigt-Radloff et al., 2012) } \\
\text { Ausbildungsaufwand: Erfahrung im Umgang mit Menschen mit kognitiven Beeinträchtigungen empfohlen (Voigt-Radloff et } \\
\text { al., 2012) } \\
\text { Benutzerfreundlichkeit: k. A. } \\
\text { Verfügbarkeit: Online } \\
\text { Verständlichkeit: Screening-Fragen sollen Verständlichkeit sicherstellen; trotzdem kommen Verständnisprobleme vor } \\
\text { (Voigt-Radloff et al., 2012) } \\
\text { Andere Sprachversionen: Englisch, Französisch, Spanisch, Chinesisch }\end{array}$ \\
\hline Validität & 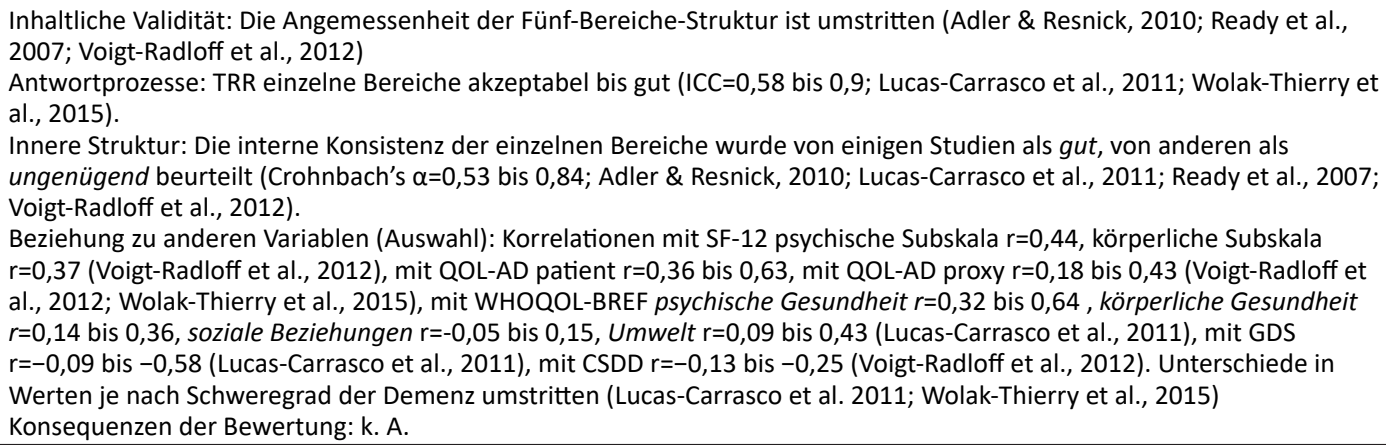 \\
\hline $\begin{array}{l}\text { Gefundene } \\
\text { Studien } \\
\text { (Autoren/ } \\
\text { Sprache/ } \\
\text { Population/ } \\
\text { Sample/Alter } \\
\text { (Durchschnitt } \\
\text { und SD)) }\end{array}$ & $\begin{array}{l}\text { Voigt-Radloff et al. 2012; Deutsch; Menschen mit Demenz }(12<\mathrm{MMSE}<26) ; \mathrm{N}=301 ; 77,3(7,2) \\
\text { Adler \& Resnick 2010; Englisch; Pflegeheimbewohner (MMSE }>12) ; \mathrm{N}=486 ; 83,8(8,2) \\
\text { Lucas-Carrasco et al. 2011; Spanisch; N=112; Menschen mit Demenz }(12<\mathrm{MMSE}<26) ; 79,3 \text { (7) } \\
\text { Ready et al. 2007; Englisch; Menschen mit Demenz (12<MMSE<26); N=67; 77,8 (6,6) } \\
\text { Wolak-Thierry et al. 2015; Französisch; Menschen mit Demenz (MMSE } \geq 10) ; 82(6)\end{array}$ \\
\hline
\end{tabular}

Anmerkungen: $C S D D=$ Cornell Scale for Depression in Dementia; GDS = Global Depression Scale; ICC = Intraclass Correlation; $N=$ Anzahl; $r=$ Spearman Correlation Coefficient; SD = Standardabweichung; TRR = Test-Retest-Reliabilität

\section{Bereiche von Lebensqualität und Wohlbefinden}

Wie erwähnt fehlen einheitliche Definitionen von Lebensqualität und Wohlbefinden. Die Assessments erfassen daher je nach zugrunde liegender Definition des Konzeptes unterschiedliche Lebensbereiche. Wenn ein Assessment zur Überprüfung oder zum Nachweis der Wirksamkeit einer Intervention angewendet werden soll, muss es die Wirkung dieser Intervention auch abbilden können. Dafür muss es die für die Klientinnen und Klienten relevanten Bereiche von Lebensqualität und Wohlbefinden erfassen, in welchen die Intervention eine Verbesserung bewirken oder eine Verschlechterung verhindern soll. Diese Bereiche werden sich je nach Klientin oder Klient, Berufsgruppe und Setting unterscheiden.

\section{Selbst- und Fremdbeurteilung von Lebensqualität und Wohlbefinden}

Lebensqualität und Wohlbefinden werden heutzutage in der Regel als subjektiv erlebte Zustände angesehen (Carr \& Higginson, 2001; Diener, 2000; Post, 2014; WHO, 2018). Assessments zur Erfassung von Lebensqualität und Wohlbefinden erfassen daher, wenn immer möglich, die Selbsteinschätzung der Befragten (Brod, Stewart \& Sands, 1999; Koller et al., 2009; Schumacher, Klaiberg \& Brähler, 2003). Gerade bei Menschen mit schwerer Demenz ist dies oft nicht möglich und stattdessen eine Fremdbeurteilung durch Angehörige und/oder Pflegefachpersonen nötig (Oppikofer, 2008). Dabei ist zu bedenken, dass sich Fremd- und Selbstbeurteilung oft deutlich unterscheiden. 
Fremdbeurteilende schätzen die Lebensqualität von Menschen mit Demenz häufig tiefer ein als die Betroffenen selbst. Dieses Phänomen zeigte sich sowohl beim SF-36 (Novella et al., 2001), dem QOL-AD (Dichter et al., 2018; Leon-Salas et al., 2011; Wolak et al., 2009) als auch dem QUALIDEM (Ettema et al., 2007a). Daher wird der Einsatz von Fremdbeurteilungsinstrumenten nur empfohlen, wenn eine Selbstbeurteilung nicht möglich ist, beispielsweise bei Menschen mit schwerer Demenz (Moniz-Cook et al., 2008).

\section{Praktikabilität und Validität der beurteilten Assessments}

Die Assessments unterscheiden sich stark in ihrer Länge, wobei EQ-5D und WHO-5 mit dem geringsten Zeitaufwand und der geringsten Belastung für die Befragten verbunden sind. Dagegen sind WHOQOLBREF und SF-36 relativ umfangreich. Außer bei der Selbstbeurteilung von Menschen mit mittelschwerer bis schwerer Demenz kam es in allen Assessments nur zu wenigen fehlenden Bewertungen. Lediglich Fragen zum Sexualleben, wie sie im WHOQOL-BREF vorkommen, wurden oft nicht beantwortet (Kalfoss et al., 2008; Lucas-Carrasco, Skevington, et al., 2011). Beim QOL$\mathrm{AD}$ fehlten in der Fremdbeurteilung oft Bewertungen zum Bereich „Geld“ (Dichter et al., 2018). Für SF-12 und WHO-5 fehlen Angaben zur Praktikabilität in der Anwendung mit Menschen mit Demenz. SF-36 und SF12 sind aufgrund ihrer Ausrichtung auf den häuslichen Alltag für den Einsatz im stationären Setting weniger geeignet (Bullinger, 2000), das QUALIDEM hingegen vor allem für dieses Setting (Ettema et al., 2007b, 2007a). Bei Menschen mit schwerer Demenz war die Durchführung von Selbstbeurteilungs-Assessments jeweils stark erschwert (Ankri et al., 2003; Baró et al., 2006; Novella et al., 2001; Tabali et al., 2012).

Für die generischen Assessments EQ-5D, NHP, SF-36 und WHOQOL-BREF wurde Evidenz für ihre Validität in der Anwendung mit älteren Menschen mit körperlichen und/oder kognitiven Einschränkungen identifiziert. Dabei wird die inhaltliche Validität und innere Struktur für den SF-36 am besten beurteilt, während für die anderen Assessments die Beurteilung widersprüchlich ausfällt. Für das NHP wurde die Test-Retest-Reliabilität von einer Studie als gut beurteilt (Tabali et al., 2012), für den EQ-5D, SF-36 und WHOQOL-BREF nur teilweise als akzeptabel (Ankri et al., 2003; Karlawish et al., 2008; Lucas-Carrasco, Skevington, et al., 2011; Naglie et al., 2006; Novella et al., 2001). Alle vier Assessments zeigen eine gute Validität betreffend die Beziehung zu anderen Variablen.

Für SF-12 und WHO-5 wurde keine aktuelle Evidenz zur Validität in der Anwendung mit Menschen mit Demenz gefunden. Insgesamt kann ihre Validität in Bezug auf Inhalt, innere Struktur und Beziehung zu anderen Variablen als akzeptabel beurteilt werden. Bezüglich der Antwortprozesse, vor allem der Test-Retest-Reliabilität, gibt es allerdings wenig Evidenz.

Für DQOL, QUALIDEM und QOL-AD waren die Ergebnisse zur inhaltlichen Validität und inneren Struktur nicht eindeutig. In Bezug auf die Antwortprozesse unterschieden sich die Assessments jedoch. So wurde die Inter-Rater-Reliabilität für die deutsche Version des QUALIDEM als gut beurteilt (Dichter et al., 2014, 2016), während sie für den QOL-AD als schwach bis mittel beurteilt wurde (Dichter et al., 2018; Leon-Salas et al., 2011; Wolak-Thierry et al., 2015). Für den DQOL liegen keine Ergebnisse vor. Auch die Test-Retest-Reliabilität wurde für das QUALIDEM als gut beurteilt (Dichter et al., 2014; Ettema et al., 2007b), während die Ergebnisse für QOL-AD und DQOL nicht eindeutig waren (Barrios et al., 2013; Leon-Salas et al., 2011; Lucas-Carrasco et al., 2011; Wolak et al., 2009; Wolak-Thierry et al., 2015). KaumeineStudieuntersuchtedie Veränderungssensitivität der Assessments. Lediglich für den SF-12 liegt hier Evidenz vor. Haibel und Kollegen (2004) beurteilten die Veränderungssensitivität der deutschen Version des SF-12 als gut in der Anwendung mit Menschen mit Ankolysierender Spondylitis.

\section{SCHLUSSFOLGERUNGEN}

Die Ergebnisse dieser Arbeit legen die Schlussfolgerung nahe, dass es kein Erfassungsinstrument gibt, das als «Goldstandard» der Erfassung von Lebensqualität und Wohlbefinden dienen kann, sondern dass eine bewusste Auswahl eines geeigneten Assessments aufgrund der Klientengruppe und des Settings erfolgen sollte. Trotzdem lassen sich grundsätzliche Empfehlungen abgeben.

Um diejenigen Bereiche von Lebensqualität und Wohlbefinden abdecken zu können, welche für ältere Menschen mit körperlichen und/oder kognitiven Einschränkungen relevant sind, ist es von Vorteil, ein Assessment zu benutzen, welches ein breites Spektrum von Lebensbereichen erfasst. Der WHOQOL-BREF scheint dies im Vergleich zu SF-36, SF-12 und NHP bei vergleichbarer Länge, Validität und Praktikabilität besser leisten zu können. Wir empfehlen daher den Einsatz des WHOQOL-BREF mit Personen, die zu einer Selbstbeurteilung in der Lage sind.

Zur Fremdbeurteilung der Lebensqualität von Menschen mit mittelschwerer bis schwerer Demenz empfehlen wir das QUALIDEM. Da es spezifisch für den Einsatz mit Pflegeheimbewohnern entwickelt wurde, ist jedoch unklar, ob es sich auch für zuhause wohnende Menschen mit Demenz eignet. Daher ist es vorerst nur für den stationären Bereich zu empfehlen. 
Zur Untersuchung gesundheitsökonomischer Fragestellungen empfehlen wir die zusätzliche Verwendung des EQ-5D. Das Assessment ist kurz, kann zur Selbst- und zur Fremdbeurteilung eingesetzt werden und verfügt über eine solide Praktikabilität und Validität in der Anwendung mit älteren Menschen mit körperlichen und/oder kognitiven Einschränkungen. Da der EQ-5D nur eine sehr enge Auswahl von Lebensbereichen abdeckt, empfehlen wir ihn jedoch nicht für die Praxis.

\section{LIMITATIONEN}

Obwohl aufgrund der Thematik dieser Arbeit davon ausgegangen werden kann, dass der größte Teil der relevanten Literatur in deutscher oder englischer Sprache vorliegt, ist es möglich, dass anderssprachige relevante Literatur nicht eingeschlossen wurde. Auch Beiträge in Büchern und «graue Literatur» wurde in der Literaturrecherche nicht berücksichtigt. Es besteht die Möglichkeit, dass aufgrund dieser Ein- und Ausschlusskriterien relevante Assessments nicht erfasst werden konnten. Aufgrund der Breite der Recherche gehen wir jedoch davon aus, dass wir diese Möglichkeit geringhalten konnten. Bei den Angaben zur Validität in den Abbildungen 4 bis 12 war es jeweils nicht möglich, alle Ergebnisse der untersuchten Studien umfassend abzubilden. Es musste eine Auswahl besonders relevanter Ergebnisse getroffen werden. Zudem kann der Heterogenität der Ergebnisse nur bedingt Rechnung getragen werden. Für eine vertiefte Auseinandersetzung mit der Validität dieser Assessments empfehlen wir, die Primärquellen hinzuzuziehen.

\section{DANK}

Diese Arbeit wurde durch das medi-Zentrum für medizinische Bildung Bern und den Schweizerischen Verband der Aktivierungsfachfrauen und -fachmänner (SVAT) finanziert.

\section{ETHISCHE PRÜFUNG, REGISTRIERUNG}

Keine ethische Prüfung notwendig: Literaturreview ohne Einbezug von Patienten/-innen

\section{INTERESSENSKONFLIKTE}

Keine.

\section{References}

Adler, E. \& Resnick, B. (2010). Reliability and validity of the dementia quality of life measure in nursing home residents. Western Journal of Nursing Research, 32 (Nursing Homes \& Residential Care [3377]), 686-704.

AERA, APA \& NCME. (2014). Standards for educational and psychological testing. Washington: American Educational Research Association.

Aguirre, E., Kang, S., Hoare, Z., Edwards, R., Orrell, M. \& Edwards, R. T. (2016). How does the EQ-5D perform when measuring quality of life in dementia against two other dementia-specific outcome measures? Quality of Life Research, 25(1), 45-49.

Aigner, M., Förster-Streffleur, S., Prause, W., Freidl, M., Weiss, M. \& Bach, M. (2006). What does the WHOQOL-Bref measure? Measurement overlap between quality of life and depressive symptomatology in chronic somatoform pain disorder. Social Psychiatry and Psychiatric Epidemiology, 41(1), 81-86.

Allgaier, A.-K., Kramer, D., Saravo, B., Mergl, R., Fejtkova, S. \& Hegerl, U. (2013). Beside the Geriatric Depression Scale: the WHO-Five Wellbeing Index as a valid screening tool for depression in nursing homes. International journal of geriatric psychiatry, 28(11), 1197-1204.

Ankri, J., Beaufils, B., Novella, J.-L., Morrone, I., Guillemin, F., Jolly, D. et al. (2003). Use of the EQ-5D among patients suffering from dementia. Journal of clinical epidemiology, 56(11), 1055-63.

Arons, A. M. M., Wetzels, R. B., Zwijsen, S., Verbeek, H., van de Ven, G., Ettema, T. P. et al. (2018). Structural validity and internal consistency of the Qualidem in people with severe dementia. International Psychogeriatrics, 30(1), 49-59.
Baró, E., Ferrer, M., Vázquez, O., Miralles, R., Pont, A., Esperanza, A. et al. (2006). Using the Nottingham Health Profile (NHP) among older adult inpatients with varying cognitive function. Quality of Life Research, 15(4), 575-585.

Barrios, H., Verdelho, A., Narciso, S., Goncalves-Pereira, M., Logsdon, R. $\&$ de Mendonca, A. (2013). Quality of life in patients with cognitive impairment: validation of the Quality of Life-Alzheimer's Disease scale in Portugal. International psychogeriatrics, 25(7), 1085-96.

Bouman, A. I. E., Ettema, T. P., Wetzels, R. B., van Beek, A. P. A., de Lange, J. \& Dröes, R. M. (2011). Evaluation of Qualidem: a dementia-specific quality of life instrument for persons with dementia in residential settings; scalability and reliability of subscales in four Dutch field surveys. International Journal of Geriatric Psychiatry, 26(7), 711-722.

Boyer, F., Novella, J.-L., Morrone, I., Jolly, D. \& Blanchard, F. (2004). Agreement between dementia patient report and proxy reports using the Nottingham Health Profile. International Journal of Geriatric Psychiatry, 19(11), 1026-1034.

Brähler, E., Mühlan, H., Albani, C. \& Schmidt, S. (2007). Teststatistische Prüfung und Normierung der Deutschen Versionen des EUROHISQOL Lebensqualität-Index und des WHO-5 Wohlbefindens-Index. Diagnostica, 53(2), 83-96.

Brettschneider, C., Konig, H.-H., Herzog, W., Kaufmann, C., Schaefert, R. \& Konnopka, A. (2013). Validity and responsiveness of the EQ-5D in assessing and valuing health status in patients with somatoform disorders. Health and quality of life outcomes, 11(101153626), 3. 
Brod, M., Stewart, A. L. \& Sands, L. (1999). Conceptualization of quality of life in dementia. Journal of Mental Health and Aging, 5 (Clinical Psychological Testing [2224]), 7-19.

Brod, M., Stewart, Sands, L. \& Walton, P. (1999). Conceptualization and measurement of quality of life in dementia: the Dementia Quality of Life Instrument (DQoL). Gerontologist, 39(1), 25-35.

Bryan, S., Hardyman, W., Bentham, P., Buckley, A. \& Laight, A. (2005). Proxy completion of EQ-5D in patients with dementia. Quality of life research : an international journal of quality of life aspects of treatment, care and rehabilitation, 14(1), 107-18.

Buchcik, J., Westenhöfer, J., Fleming, M. \& Martin, C. R. (2017). Is health-related quality of life the same for elderly polish migrants, Turkish migrants and German natives? Testing the reliability and construct validity of the Sf-36 health survey in a cross-cultural comparison. Cogent Psychology, 4(1), 1-15.

Bullinger,M.(2000).ErfassungdergesundheitsbezogenenLebensqualität mit dem SF-36-Health Survey. BundesgesundheitsblattGesundheitsforschung-Gesundheitsschutz, 43(3), 190-197.

Bullinger, M., Kohlmann T, Nantke J, van den Bussche H, Dodt B, Dunkelberg S et al. (2003). SF-36 Health Survey in rehabilitation research. Findings from the North German Network for Rehabilitation Research, NVRF, within the rehabilitation research funding programme. Rehabilitation, 42(4), 218-225.

Bundesamt für Gesundheit. (2013). Die gesundheitspolitischen Prioritäten des Bundesrates. Bern: Bundesamt für Gesundheit.

Bundesamt für Statistik. (2015). Szenarien zur Bevölkerungsentwicklung der Kantone der Schweiz 2015-2045-Ständige Wohnbevölkerung nach Kantonen gemäss 3 Szenarien. Neuchâtel: Bundesamt für Statistik. Zugriff am 8.4.2018. Verfügbar unter: https://www.bfs. admin.ch/bfsstatic/dam/assets/350324/master

Bundesministerium für Arbeit, Soziales, Gesundheit und Konsumentenschutz. (2019). Gesundheitsziele Österreich. Zugriff am 15.2.2019. Verfügbar unter: https://gesundheitsziele-oesterreich.at/

Carr, A. J. \& Higginson, I. J. (2001). Measuring quality of life: Are quality of life measures patient centred? BMJ: British Medical Journal, 322(7298), 1357.

Coucill, W., Bryan, S., Bentham, P., Buckley, A. \& Laight, A. (2001). EQ-5D in patients with dementia: an investigation of inter-rater agreement. Medical care, 39(8), 760-71.

Daig, I. \& Lehmann, A. (2007). Verfahren zur Messung der Lebensqualität. Zeitschrift für medizinische Psychologie, 16(1, 2), $5-23$.

Diaz-Redondo, A., Rodriguez-Blazquez, C., Ayala, A., MartinezMartin, P. \& Forjaz, M. J. (2014). EQ-5D rated by proxy in institutionalized older adults with dementia: Psychometric pros and cons. Geriatrics \& Gerontology International, 14(2), 346-353.

Dichter, M., Bartholomeyczik, S., Nordheim, J., Achterberg, W. \& Halek, M. (2011). Validity, reliability, and feasibility of a quality of life questionnaire for people with dementia. Zeitschrift fur Gerontologie und Geriatrie, 44(6), 405-10.

Dichter, M. N., Dortmann, O., Halek, M., Meyer, G., Holle, D., Nordheim, J. et al. (2013). Scalability and internal consistency of the German version of the dementia-specific quality of life instrument QUALIDEM in nursing homes - a secondary data analysis. Health \& Quality of Life Outcomes, 11(1), 91-91.
Dichter, M. N., Schwab, C. G. G., Meyer, G., Bartholomeyczik, S., Dortmann, O. \& Halek, M. (2014). Measuring the quality of life in mild to very severe dementia: testing the inter-rater and intra-rater reliability of the German version of the QUALIDEM. International Psychogeriatrics, 26(5), 825-836.

Dichter, M. N., Wolschon, E.-M., Meyer, G. \& Kopke, S. (2016). Cross-cultural adaptation of the German version of the Quality of Life in Alzheimer's Disease scale - Nursing Home version (QoLAD NH). International psychogeriatrics, 28(8), 1399-400.

Dichter, M. N., Wolschon, E.-M., Schwab, C. G., Meyer, G. \& Köpke, S. (2018). Item distribution and inter-rater reliability of the German version of the quality of life in Alzheimer's disease scale (QoL$\mathrm{AD})$ proxy for people with dementia living in nursing homes. BMC geriatrics, 18(1), 145.

Diener, E. (2000). Subjective well-being: The science of happiness and a proposal for a national index. American psychologist, 55(1), 34.

Diener, E., Emmons, R. A., Larsen, R. J. \& Griffin, S. (1985). The satisfaction with life scale. Journal of personality assessment, 49(1), 71-75.

Dronavalli, M. \& Thompson, S. C. (2015). A systematic review of measurement tools of health and well-being for evaluating community-based interventions. Journal of Epidemiology and Community Health, 69(8), 805-815.

Easton, T., Milte, R., Crotty, M. \& Ratcliffe, J. (2018). An empirical comparison of the measurement properties of the EQ-5D5L, DEMQOL-U and DEMQOL-Proxy-U for older people in residential care. Quality of Life Research, 27(5), 1283-1294.

Englbrecht, M., Alten, R., Aringer, M., Baerwald, C. G., Burkhardt, H., Eby, N. et al. (2017). Validation of standardized questionnaires evaluating symptoms of depression in rheumatoid arthritis patients: approaches to screening for a frequent yet underrated challenge. Arthritis care \& research, 69(1), 58-66.

Ettema, T. P., Dröes, R., de Lange, J., Mellenbergh, G. J. \& Ribbe, M. W. (2007b). QUALIDEM: development and evaluation of a dementia specific quality of life instrument. Scalability, reliability and internal structure. International Journal of Geriatric Psychiatry, 22(6), 549-556.

Ettema, T. P., Dröes, R., de Lange, J., Mellenbergh, G. J. \& Ribbe, M. W. (2007a). QUALIDEM: development and evaluation of a dementia specific quality of life instrument--validation. International Journal of Geriatric Psychiatry, 22(5), 424-430.

EuroQol, G. (1990). EuroQol - a new facility for the measurement of health-related quality of life. Health policy (Amsterdam, Netherlands), 16(3), 199.

Fawcett, A. L. (2013). Principles of assessment and outcome measurement for occupational therapists and physiotherapists: theory, skills and application. John Wiley \& Sons.

Frosch, D., Porzsolt, F., Heicappell, R., Kleinschmidt, K., Schatz, M., Weinknecht, S. et al. (2001). Comparison of German language versions of the QWB-SA and SF-36 evaluating outcomes for patients with prostate disease. Quality of life research: an international journal of quality of life aspects of treatment, care and rehabilitation, 10(2), 165-73.

Glasgow, R. E., Magid, D. J., Beck, A., Ritzwoller, D. \& Estabrooks, P. A. (2005). Practical clinical trials for translating research to 
practice: design and measurement recommendations. Medical care, 43, 551-557.

Gräske, J., Verbeek, H., Gellert, P., Fischer, T., Kuhlmey, A. \& Wolf-Ostermann, K. (2014). How to measure quality of life in shared-housing arrangements? A comparison of dementia-specific instruments. Quality of Life Research, 23(2), 549-559.

Günther, O. H., Roick, C., Angermeyer, M. C. \& König, H.-H. (2008). The responsiveness of EQ-5D utility scores in patients with depression: A comparison with instruments measuring quality of life, psychopathology and social functioning. Journal of Affective Disorders, 105(1-3), 81-91.

Gunzelmann, T., Albani, C., Beutel, M. \& Brähler, E. (2006). Die subjektive Gesundheit älterer Menschen im Spiegel des SF-36: Normwerte aus einer bevölkerungsrepräsentativen Erhebung. Zeitschrift für Gerontologie und Geriatrie, 39(2), 109-119.

Haibel, H., Niewerth, M., Brandt, J., Rudwaleit, M., Listing, J., Sieper, J. et al. (2004). [Measurement of quality of life in patients with active ankylosing spondylitis being treated with infliximab-a comparison of SF-36 and SF-12]. Messung der Lebensqualität unter Therapie mit Infliximab bei Patienten mit aktiver ankylosierender Spondylitis. Ein Vergleich von SF-36 und SF-12., 63(5), 393-401.

Hammell, K. W. (2006). Perspectives on disability \& rehabilitation: contesting assumptions, challenging practice. Edinburgh: Churchill Livingstone.

Heun, R., Bonsignore, M., Barkow, K. \& Jessen, F. (2001). Validity of the five-item WHO Well-Being Index (WHO-5) in an elderly population. European Archives of Psychiatry and Clinical Neuroscience, 251(S2), 27-31.

Hinz, A., Klaiberg, A., Schumacher, J. \& Brahler, E. (2003). Zur psychometrischen Qualität des Lebensqualitätsfragebogens Nottingham Health Profile (NHP) in der Allgemeinbevölkerung. Psychotherapie- Psychosomatik-- Medizinische - Psychologie, 53(8), 353-358.

Hoe, J., Katona, C., Roch, B. \& Livingston, G. (2005). Use of the QOL$\mathrm{AD}$ for measuring quality of life in people with severe dementia - the LASER-AD study. Age and ageing, 34(2), 130-5.

Holland, R., Smith, R. D., Harvey, I., Swift, L. \& Lenaghan, E. (2004). Assessing quality of life in the elderly: a direct comparison of the EQ-5D and AQoL. Health economics, 13(8), 793-805.

Hunt, S. M., McKenna, S. P., McEwen, J., Williams, J. \& Papp, E. (1981). The Nottingham Health Profile: subjective health status and medical consultations. Social Science \& Medicine. Part A: Medical Psychology \& Medical Sociology, 15(3), 221-229.

Hylla, J., Schwab, C. G. G., Isfort, M., Halek, M. \& Dichter, M. N. (2016). Interne Konsistenz und Konstruktvalidität des Quality of Life in Alzheimer's Disease (QoLAD) proxy Instruments: Eine Sekundärdatenanalyse. Pflege, 29(4), 183-191.

Jakobsson, U. (2007). Using the 12-item Short Form health survey (SF$12)$ to measure quality of life among older people. Aging Clinical \& Experimental Research, 19(6), 457-464.

Jakobsson, U., Westergren, A., Lindskov, S. \& Hagell, P. (2012). Construct validity of the SF-12 in three different samples. Journal of Evaluation in Clinical Practice, 18(3), 560-566.
Kalfoss, M. H., Low, G. \& Molzahn, A. E. (2008). The suitability of the WHOQOL-BREF for Canadian and Norwegian older adults. World Health Organization Quality of Life Measure Abbreviated Version. European Journal of Ageing, 5(1), 77-89.

Karlawish, J. H., Zbrozek, A., Kinosian, B., Gregory, A., Ferguson, A. \& Glick, H. A. (2008). Preference-based quality of life in patients with Alzheimer's disease. Alzheimer's \& dementia : the journal of the Alzheimer's Association, 4(3), 193-202.

Koller, M., Neugebauer, E. A. M., Augustin, M., Büssing, A., Farin, E., Klinkhammer-Schalke, M. et al. (2009). Die Erfassung von Lebensqualität in der Versorgungsforschungkonzeptuelle, methodische und strukturelle Voraussetzungen. Das Gesundheitswesen, 71(12), 864-872.

Kunz, S. (2010). Psychometric properties of the EQ-5D in a study of people with mild to moderate dementia. Quality of Life Research: An International Journal of Quality of Life Aspects of Treatment, Care \& Rehabilitation, 19, 425-434. https://doi.org/10.1007/ s11136-010-9600-1.

Leon-Salas, B., Logsdon, R. G., Olazaran, J., Martinez-Martin, P. \& The Msu-Adru. (2011). Psychometric properties of the Spanish QoL-AD with institutionalized dementia patients and their family caregivers in Spain. Aging \& mental health, 15(6), 775-83.

Logsdon, R. G., Gibbons, L. E., McCurry, S. M. \& Teri, L. (1999). Quality of life in Alzheimer's disease: patient and caregiver reports. Journal of Mental health and Aging, 5, 21-32.

Lucas-Carrasco, R., Gomez-Benito, J., Rejas, J. \& Brod, M. (2011). The Spanish version of the dementia quality of life questionnaire: A validation study. Aging \& Mental Health, 15(4), 482-489.

Lucas-Carrasco, R., Skevington, S. M., Gómez-Benito, J., Rejas, J. \& March, J. (2011). Using the WHOQOL-BREF in persons with dementia: a validation study. Alzheimer Disease \& Associated Disorders, 25(4), 345-351.

Maurischat, C., Ehlebracht-Konig, I., Kuhn, A. \& Bullinger, M. (2006). Factorial validity and norm data comparison of the Short Form 12 in patients with inflammatory-rheumatic disease. Rheumatology international, 26(7), 614-21.

Maurischat, C., Herschbach, P., Peters, A. \& Bullinger, M. (2008). Factorial validity of the Short Form 12 (SF-12) in patients with diabetes mellitus, 14.

Moniz-Cook, E., Vernooij-Dassen, M., Woods, R., Verhey, F., Chattat, R., Vugt, M. D. et al. (2008). A European consensus on outcome measures for psychosocial intervention research in dementia care. Aging \& Mental Health, 12(1), 14-29.

Morfeld, M., Bullinger, M., Nantke, J. \& Brähler, E. (2005). Die Version 2.0 des SF-36 Health Survey - Ergebnisse einer bevölkerungsrepräsentativen Studie. Sozial- und Präventivmedizin SPM, 50(5), 292-300.

Morfeld, M., Dietsche, S., Bürger, W. \& Koch, U. (2003). Der SF-12 - Das Problem der Missing Data. Diagnostica, 49(3), 129-135. https://doi.org/10.1026//0012-1924.49.3.129.

Muller-Nordhorn, J. (2004). Comparison of the short form (SF)-12 health status instrument with the SF-36 in patients with coronary heart disease. Heart, 90(5), 523-527. 
Naglie, G., Tomlinson, G., Tansey, C., Irvine, J., Ritvo, P., Black, S. E. et al. (2006). Utility-based quality of life measures in Alzheimer's disease. Quality of Life Research, 15(4), 631-643.

Naumann, V. J. \& Byrne, G. J. A. (2004). WHOQOL-BREF as a measure of quality of life in older patients with depression. International Psychogeriatrics, 16(2), 159-173.

Novella, J. L., Jochum, C., Ankri, J., Morrone, I., Jolly, D. \& Blanchard, F. (2001). Measuring general health status in dementia: Practical and methodological issues in using the SF-36. Aging Clinical and Experimental Research, 13(5), 362-369.

Oppikofer, S. (2008). Lebensqualität bei Demenz: eine Bestandesaufnahme, Sichtung und Dokumentation bestehender Instrumente zur Messung von Lebensqualität bei Menschen mit schwerer Demenz. Zürich: Universität Zürich, Zentrum für Gerontologie.

Orgeta, V., Edwards, R. T., Hounsome, B., Orrell, M. \& Woods, B. (2015). The use of the EQ-5D as a measure of health-related quality of life in people with dementia and their carers. Quality of life research : an international journal of quality of life aspects of treatment, care and rehabilitation, 24(2), 315-24.

Post, M. (2014). Definitions of Quality of Life: What Has Happened and How to Move On. Topics in Spinal Cord Injury Rehabilitation, 20(3), 167-180. https://doi.org/10.1310/sci2003-167.

Power, M. (2003). Development of a common instrument for quality of life. In A. Nosikov \& C. Gudex (Hrsg.), EUROHIS: Developing common instruments for health surveys (S. 145-164). Amsterdam: IOS.

Ready, R. E., Ott, B. R. \& Grace, J. (2007). Factor structure of patient and caregiver ratings on the dementia quality of life instrument. Neuropsychology, development, and cognition. Section B, Aging, neuropsychology and cognition, 14(2), 144-54.

Schneider, C. B., Pilhatsch, M., Rifati, M., Jost, W. H., Wodarz, F., Ebersbach, G. et al. (2010). Utility of the WHO-five well-being index as a screening tool for depression in Parkinson's disease. Movement disorders, 25(6), 777-783.

Scholz, M., Müller, E., Kröhne, U., Böcker, M., Forkmann, T. \& Wirtz, M. (2013). Konfirmatorische Prüfung der dimensionalen Struktur des Oswestry Disability Index, des SF-12 und der HADS-D bei Rehabilitanden mit muskuloskelettalen Erkrankungen. Die Rehabilitation, 53(02), 118-123.

Schumacher, J., Klaiberg, A. \& Brähler, E. (Hrsg.). (2003). Diagnostische Verfahren zu Lebensqualität und Wohlbefinden. Göttingen: Hogrefe.

Seymour, D. G., Ball, A. E., Russell, E. M., Primrose, W. R., Garratt, A. M. \& Crawford, J. R. (2001). Problems in using health survey questionnaires in older patients with physical disabilities. The reliability and validity of the SF-36 and the effect of cognitive impairment. Journal of Evaluation in Clinical Practice, 7(4), 411418.

Simon, A., Berwig, M. \& Heinrich, S. (2013). Quality of life in nursing homes following the group-living principle: A pilot study measuring the quality of life from the subjective perspective of dependent-living elderly. Zeitschrift fur Gerontologie und Geriatrie, 46(6), 556-562.
Skevington, S. M., Lotfy, M. \& O'Connell, K. A. (2004). The World Health Organization's WHOQOL-BREF quality of life assessment: Psychometric properties and results of the international field trial. A Report from the WHOQOL Group. Quality of Life Research, 13(2), 299-310.

Sonntag, M., Konig, H.-H. \& Konnopka, A. (2015). The responsiveness of the EQ-5D and time trade-off scores in schizophrenia, affective disorders, and alcohol addiction. Health and quality of life outcomes, 13(101153626), 114.

Statistik Austria. (2018). Datenquelle: Bevölkerung zum Jahresanfang 1952 bis 2101. Zugriff am 14.2.2019. Verfügbar unter: http:// statcube.at/statistik.at/ext/statcube/jsf/tableView/tableView.xhtml.

Statistisches Bundesamt. (2015). Koordinierte Bevölkerungsvorausberechnung für Deutschland. Zugriff am 14.2.2019. Verfügbar unter: https://service.destatis.de/bevoelkeru ngspyramide/\#! $\mathrm{y}=2045 \& \mathrm{v}=6$.

Tabali, M, Jeschke E, Dassen T, Ostermann T, Heinze C, Tabali, Manuela et al. (2012). The Nottingham Health Profile: a feasible questionnaire for nursing home residents? International Psychogeriatrics, 24(3), 416-424.

Topp, C. W., Østergaard, S. D., Søndergaard, S. \& Bech, P. (2015). The WHO-5 Well-Being Index: A Systematic Review of the Literature. Psychotherapy \& Psychosomatics, 84(3), 167-176.

Tunis, S. R., Stryer, D. B. \& Clancy, C. M. (2003). Practical clinical trials: increasing the value of clinical research for decision making in clinical and health policy. Jama, 290(12), 1624-1632.

Voigt-Radloff, S., Leonhart, R., Schutzwohl, M., Jurjanz, L., Reuster, T., Gerner, A. et al. (2012). Dementia quality of life instrumentConstruct and concurrent validity in patients with mild to moderate dementia. European Journal of Neurology, 19, 376-384.

Ware, J. E., Kosinski, M. \& Keller, S. D. (1996). A 12-Item ShortForm Health Survey: construction of scales and preliminary tests of reliability and validity. Medical care, 34(3), 220-233.

Ware, J. E. \& Sherbourne, C. D. (1992). The MOS 36-item short-form health survey (SF-36): I. Conceptual framework and item selection. Medical care, 473-483.

Watson, D., Clark, L. A. \& Tellegen, A. (1988). Development and validation of brief measures of positive and negative affect: the PANAS scales. Journal of personality and social psychology, 54(6), 1063.

WHO. (2018). WHO I WHOQOL: Measuring Quality of Life. WHO. Zugriff am 18.4.2018. Verfügbar unter: http://www.who.int/ healthinfo/survey/whoqol-qualityoflife/en/\#.

WHOQOL-Group. (1998). Development of the World Health Organization WHOQOL-BREF quality of life assessment. Psychological Medicine, 28(3), 551-558.

Wirtz, M. A., Morfeld, M., Glaesmer, H. \& Brähler, E. (2018). Konfirmatorische Prüfung der Skalenstruktur des SF-12 Version 2.0 in einer deutschen bevölkerungsrepräsentativen Stichprobe. Diagnostica, 64(2), 84-96.

Wolak, A., Novella, J., Drame, M., Guillemin, F., DiPollina, L., Ankri J et al. (2009). Transcultural adaptation and psychometric validation of a French-language version of the QoL-AD. Aging \& Mental Health, 13(4), 593-600. 
Wolak-Thierry, A., Novella, J.-L., Barbe, C., Morrone, I., Mahmoudi, R. \& Jolly, D. (2015). Comparison of QoL-AD and DQoL in elderly with Alzheimer's disease. Aging \& Mental Health, 19(3), 274-278.

World Health Organization Regional Office for Europe. (1998). Wellbeing measures in primary health care: The DepCare project. Consensus meeting. Stockholm: WHO Regional Office. 